Cite this: Soft Matter, 2014, 10, 2785

\title{
Force spectroscopy of polymer desorption: theory and molecular dynamics simulations
}

\author{
Jarostaw Paturej, ${ }^{\text {ab }}$ Johan L. A. Dubbeldam, ${ }^{\text {C Vakhtang G. Rostiashvili, }}{ }^{d}$ \\ Andrey Milchev ${ }^{\mathrm{de}}$ and Thomas A. Vilgis ${ }^{\mathrm{d}}$
}

Forced detachment of a single polymer chain, strongly adsorbed on a solid substrate, is investigated by two complementary methods: a coarse-grained analytical dynamical model, based on the Onsager stochastic equation, and Molecular Dynamics (MD) simulations with a Langevin thermostat. The suggested approach makes it possible to go beyond the limitations of the conventional Bell-Evans model. We observe a series of characteristic force spikes when the pulling force is measured against the cantilever displacement during detachment at constant velocity $v_{c}$ (displacement control mode) and find that the average magnitude of this force increases as $v_{c}$ increases. The probability distributions of the pulling force and the endmonomer distance from the surface at the moment of the final detachment are investigated for different adsorption energies $\varepsilon$ and pulling velocities $v_{\mathrm{c}}$. Our extensive MD simulations validate and support the main theoretical findings. Moreover, the simulations reveal a novel behavior: for a strong-friction and massive cantilever the force spike pattern is smeared out at large $v_{c}$. As a challenging task for experimental bio-polymer sequencing in future we suggest the fabrication of a stiff, super-light, nanometer-sized AFM probe.

Received 10th October 2013 Accepted 8th January 2014

DOI: $10.1039 / c 3 s m 52618 f$

www.rsc.org/softmatter limitations with regard to the mechanochemistry of weak interactions in the lower piconewton regime.

The method of dynamic force spectroscopy (DFS) is used to probe the force-extension relationship, rupture force distribution, and the force vs. loading rate dependence for singlemolecule bonds or for more complicated multiply bonded attachments. Historically, the first theoretical interpretation of DFS has been suggested in the context of single cell adhesion by Bell $^{12}$ and developed by Evans. ${ }^{13-15}$ The consideration has been based on the semi-phenomenological Arrhenius relationship which describes surface detachment under time-dependent pulling force, $f=r_{1}$, with $r_{1}$ being the loading rate. It was also assumed that the effective activation energy, $E_{\mathrm{b}}(f)$, may be approximated by a linear function of the force, i.e., $E_{\mathrm{b}}(f)=E_{\mathrm{b}}^{(0)}-$ $x_{\beta} f$. Here $x_{\beta}$ is the distance between the bonded state and the transition state where the activation barrier is located. The resulting Bell-Evans (BE) equation then gives the mean detachment force as a function of temperature $T$ and loading rate $r_{1}$, i.e., $f=\frac{k_{\mathrm{B}} T}{x_{\beta}} \ln \left(\frac{r_{1} x_{\beta}}{k_{\mathrm{B}} T \kappa_{0}}\right)$, where $\kappa_{0}$ is the desorption rate in the absence of applied pulling force.

As one can see from this $\mathrm{BE}$ equation, the simple surmounting of the BE-activation barrier results in a linear dependence of the detachment force on the logarithm of the loading rate, provided one uses the applied force as a governing parameter in the detachment process (i.e., working in an isotensional ensemble when $f$ is controlled and the distance $D$ from the substrate to the clamped end-monomer of the polymer
${ }^{a}$ Department of Chemistry, University of North Carolina, Chapel Hill, NC 27599, USA.
E-mail: paturej@live.unc.edu
${ }^{b}$ Institute of Physics, University of Szczecin, Wielkopolska 15, 70451 Szczecin, Poland
${ }^{c}$ Delft University of Technology, 2628CD Delft, The Netherlands
${ }^{d}$ Max Planck Institute for Polymer Research, 10 Ackermannweg, 55128 Mainz,
Germany
${ }^{e}$ Institute of Physical Chemistry, Bulgarian Academy of Sciences, 1113 Sofia, Bulgaria 
chain fluctuates). For multiply bonded attachments the interpretation problem based on this equation becomes more complicated since a non-linear $f$-ln $r_{1}$ relationship is observed. ${ }^{\mathbf{1 6}}$ In this case chain detachment involves passage over a cascade of activation barriers. For example, Merkel et al. ${ }^{16}$ suggested that the net rate of detachments can be approximated by a reciprocal sum of characteristic times, corresponding to jumps over the single barriers. In particular, regarding the detachment of biotinstreptavidin single bonds, it was suggested that two consecutive barriers might be responsible for the desorption process.

A simple example of multiply bonded bio-assembly is presented by a singe-stranded DNA (ssDNA) macromolecule, strongly adsorbed on the graphite substrate. The force-induced desorption (or peeling) of this biopolymer has been studied analytically and by means of Brownian dynamics (BD) simulations by Jagota et al. ${ }^{17-20}$ In ref. 17 the equilibrium statistical thermodynamics of ssDNA force-induced desorption under force control (FC) and displacement control (DC) have been investigated. In the latter case one works in an isometric ensemble where $D$ is controlled and $f$ fluctuates. It has been demonstrated that the force response under DC exhibits a series of spikes which carry information about the underlying base sequence of ssDNA. The Brownian dynamics (BD) simulations ${ }^{\mathbf{1 8}}$ confirmed the existence of such force spikes in the forcedisplacement curves under DC.

The nonequilibrium theory of forced desorption has been developed by Kreuzer et al. ${ }^{\mathbf{2 1 - 2 3}}$ on the basis of the master equation approach for the cases of constant velocity and forceramp modes in an AFM experiment. The authors assumed that the detachment of individual monomers represents a fast process as compared to the removal of all monomers. This justifies a two-state model where all monomers either remain on the substrate or leave it abruptly. The corresponding transition rates (which constitute a necessary input in the master equation approach) must satisfy a detailed balance. As a result of the master equation solution, the authors obtained a probability distribution of detachment heights (i.e., distances between the cantilever tip and the substrate) as well as an average detachment height as a function of the pulling velocity.

Irrespective of all these efforts, a detailed theoretical interpretation of the dynamic force spectroscopy experiments is still missing. For example, in terms of Kramers reaction-rate theory $^{\mathbf{2 4}}$ the Arrhenius-like BE model holds only when the effective activation energy $E_{\mathrm{b}}(f) \gg k_{\mathrm{B}} T$. On the other hand, it is clear that for large forces (which we experience in AFM), the case when $E_{\mathrm{b}}(f) \approx k_{\mathrm{B}} T$ occurs fairly often. In this common case the general approach, based on the BE-model, becomes questionable. Besides, it can be shown, ${ }^{25}$ that the activation energy $v s$. force dependence, $E_{\mathrm{b}}(f)$, is itself a nonlinear function, so that the conventional BE-model, based on the linear approximation, $E_{\mathrm{b}}(f) \approx E_{\mathrm{b}}^{(0)}-x_{\beta} f$, should be limited to small forces. Moreover, the Arrhenius-like relationship for the detachment rate, which was used in the BE-model, is a consequence of a saddle-point approximation for the stationary solution of the Fokker-Planck equation. ${ }^{24}$ This contradicts the typical loading regimes, used in experiments, where the applied force or distance grows linearly with time.
The present paper is devoted to the theoretical investigation of a single molecule desorption dynamics and aimed at interpretation of AFM- or LOT-based dynamic force spectroscopy in the DC constant-velocity mode. The organization of the paper is as follows: in Section 2 we give the equilibrium theory of detachment for the case of strong polymer adsorption. The mean force (measured at the cantilever tip) versus displacement diagram is discussed in detail. In particular, the characteristic force "spikes" structure (which was first discussed in ref. 17 and 18) can be clearly seen. In Section 3 we give a dynamical version of the detachment process. Our approach rests on construction of general free energy functions, depending on coarse-grained variables, which govern the non-linear response and structural bonding changes in the presence of external forces. The corresponding free-energy-based stochastic equations (known as Onsager equations ${ }^{\mathbf{2 6}}$ ) are derived and solved numerically. This solution makes it possible to provide not only force-displacement diagrams and the ensuing dependence on the cantilever displacement velocity $v_{\mathrm{c}}$ but also the detachment force probability distribution function (PDF). In Section 4 the main theoretical results are then checked against extensive Molecular Dynamics (MD) simulations. A brief discussion of results is offered in Section 5 .

\section{Equilibrium theory in the strong adsorption case}

Recently we suggested a theory of the force-induced polymer desorption (for relatively weak adsorption energy) in the isotensional $^{27,28}$ and isometric ${ }^{29}$ equilibrium ensembles supported by extensive Monte Carlo (MC) simulations. In the former case, the fraction of adsorbed monomers changes abruptly (undergoes a jump) when one varies the adsorption energy or the external pulling force. In the second case, the order parameter varies steadily upon changing the height of the AFMtip, even though the phase transition is still of first order. The total phase diagram in terms of adsorption energy-pulling force or adsorption energy-end-monomer height has been discussed theoretically and in terms of MC simulations.

On the other hand, the AFM experiments deal with relatively strong forces (20 pN-10 nN (ref. 1)) so that in the case of a single molecule desorption experiment only a really strong adsorption energy is essential. This limit has been discussed in the recent papers by Jagota et al. ${ }^{17-20}$ and Kreuzer et al. ${ }^{21-23}$ Here we consider this problem in a slightly more general form. In doing so we distinguish between two different models: with frictionless- and strong-friction substrates, as indicated in Fig. 1.

\subsection{Frictionless substrate}

This case has been considered in ref. 17, 18 and 21-23 and is based on the assumption that the force resisting sliding is sufficiently small, i.e., the cantilever tip and the contact point $c$ are both placed along the same $z$-axis (see Fig. 1 (left panel)). The total partition function for a fixed cantilever distance $D$, i.e., $\Xi_{\text {tot }}(D)$, is a product of partition functions of the adsorbed part, $\Xi_{\text {ads }}(n)$, of the desorbed portion (a stretched polymer portion), 

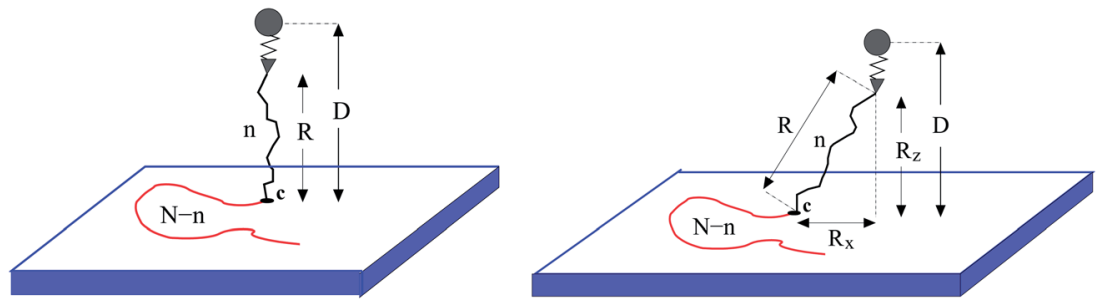

Fig. 1 Principal scheme of a single molecule forced desorption experiment based on the AFM: (left panel) There is no friction between the adsorbed portion and substrate. (right panel) Strong friction case.

$\Xi_{\mathrm{pol}}(n, R)$, and of the cantilever itself, $\Xi_{\mathrm{can}}(D-R)$, where $n$ is the number of desorbed polymer segments, and $R$ denotes the distance between the clamped end of this desorbed portion and the substrate. As a result,

$$
\Xi_{\mathrm{tot}}(D)=\sum_{n=0}^{N} \Xi_{\mathrm{ads}}(n) \int_{0}^{b n} \mathrm{~d} R \Xi_{\mathrm{pol}}(n, R) \theta(D-R) \Xi_{\mathrm{can}}(D-R),
$$

where the integration interval, $0<R<b n$, and the step-function, $\theta(D-R)$, imply that restrictions, $R<b n$ and $R<D$, should be applied simultaneously. In this representation $D$ is the control variable (which is monitored by the corresponding AFM operating mode) whereas $n$ and $R$ are coarse-grained dynamic variables which should be integrated (in our case, an integral over $R$, and summation over $n$ ) out. Moreover, if we introduce the function

$$
\min (b n, D)=\left\{\begin{array}{cl}
b n & \text { for } b n<D \\
D & \text { for } D<b n
\end{array}\right.
$$

then eqn (1) can be rewritten as

$$
\Xi_{\mathrm{tot}}(D)=\sum_{n=0}^{N} \Xi_{\mathrm{ads}}(n) \int_{0}^{\min (b n, D)} \mathrm{d} R \Xi_{\mathrm{pol}}(n, R) \Xi_{\mathrm{can}}(D-R)
$$
form

In the strong adsorption regime, $\Xi_{\text {ads }}(n)$ attains a simple

$$
\Xi_{\mathrm{ads}}(n)=\exp [\varepsilon(N-n)],
$$

where $\varepsilon$ is the dimensionless adsorption energy (i.e. energy per $k_{\mathrm{B}} T$ ). The cantilever manifests itself as a harmonic spring with a spring constant $k_{\mathrm{c}}$, i.e., the corresponding partition function reads

$$
\Xi_{\mathrm{can}}(D-R)=\exp \left[-\frac{k_{\mathrm{c}}}{2 k_{\mathrm{B}} T}(D-R)^{2}\right]
$$

Finally, we derive the partition function of the desorbed part of the polymer as a function of the dynamic variables $n$ and $R$, based on the Freely Jointed Bond Vector (FJBV) model. ${ }^{30,31}$ The corresponding calculations using the Legendre transformation are relegated to Appendix A. Thus, the polymer partition function takes the form

$$
\Xi_{\text {pol }}(n, R)=\exp [n \mathscr{S}(\tilde{f})]
$$

where $\mathscr{G}(x)=\ln [\sinh (x) / x]+1-x \operatorname{coth}(x)$. In eqn (6) the dimensionless force $\tilde{f} \stackrel{\text { def }}{=} b f / k_{\mathrm{B}} T$ should be expressed in terms of $R / b n$ as follows: $\tilde{f}=\mathscr{L}^{-1}(R / b n)$, where $\mathscr{L}^{-1}(x)$ denotes the inverse of the Langevin function $\mathscr{L}(x) \equiv \operatorname{coth}(x)-1 / x$.

By making use of eqn (4)-(6), the total partition function given by eqn (1) reads

$$
\begin{aligned}
\Xi_{\mathrm{tot}}(D)= & \sum_{n=0}^{N} \int_{0}^{\min (n b, D)} \mathrm{d} R \exp [\varepsilon(N-n)] \exp [n \mathscr{G}(\tilde{f})] \\
& \times \exp \left[-\frac{k_{\mathrm{c}}}{2 k_{\mathrm{B}} T}(D-R)^{2}\right]
\end{aligned}
$$

The corresponding effective free energy function in terms of $n$ and $R$ reads

$$
\mathscr{F}(n, R)=-k_{\mathrm{B}} T \varepsilon(N-n)-k_{\mathrm{B}} T n \mathscr{E}(\tilde{f})+\frac{k_{\mathrm{c}}}{2}(D-R)^{2}
$$

In the limit of a very stiff cantilever, $k_{\mathrm{c}} b^{2} / k_{\mathrm{B}} T \gg 1$, the cantilever partition function approaches a $\delta$-function: ${ }^{21}$

$$
\Xi_{\mathrm{can}}(D-R)=\exp \left[-\frac{k_{\mathrm{c}}}{2 k_{\mathrm{B}} T}(D-R)^{2}\right] \rightarrow\left(2 \pi k_{\mathrm{B}} T / k_{\mathrm{c}}\right)^{1 / 2} \delta(D-R),
$$

and eqn (7) takes the form

$$
\Xi_{\text {tot }}(D)=\sum_{n=0}^{N} \exp [\varepsilon(N-n)] \exp [n \mathscr{G}(\tilde{f})] \theta(n b-D),
$$

where $\tilde{f}=\mathscr{L}^{-1}(D / b n)$ and the step-function $\theta(b n-D)$ ensures that the condition $b n>D$ holds. It is this very stiff cantilever limit that was considered in ref. 17 and 18.

For the isometric ensemble, i.e., in the $D$-ensemble, the average force $\left\langle f_{z}\right\rangle$, measured by the AFM experiment, is given by

$$
\begin{aligned}
\left\langle f_{z}\right\rangle= & -k_{\mathrm{B}} T \frac{\partial}{\partial D} \ln \Xi_{\mathrm{tot}}(D)=\frac{k_{\mathrm{c}}}{\Xi_{\mathrm{tot}}(D)} \sum_{n=0}^{N} \exp [\varepsilon(N-n)] \\
& \times \int_{0}^{\min (b n, D)} \mathrm{d} R(D-R) \exp [n \mathscr{G}(\tilde{f})] \exp \left[-\frac{k_{\mathrm{c}}}{2 k_{\mathrm{B}} T}(D-R)^{2}\right],
\end{aligned}
$$

where $\Xi_{\text {tot }}(D)$ is given by eqn (7).

The numerical results, which follow from eqn (11), are shown in Fig. 2. One can immediately see the "sawtooth"-, or force-spikes structure in the force-displacement diagram as it was also found by Jagota et $a .^{17}$ in the limit of a very stiff cantilever. Physically, spikes correspond to the reversible transitions $n \rightleftarrows n+1$, during which the release of the polymer stretching energy is balanced by the adsorption energy. The 

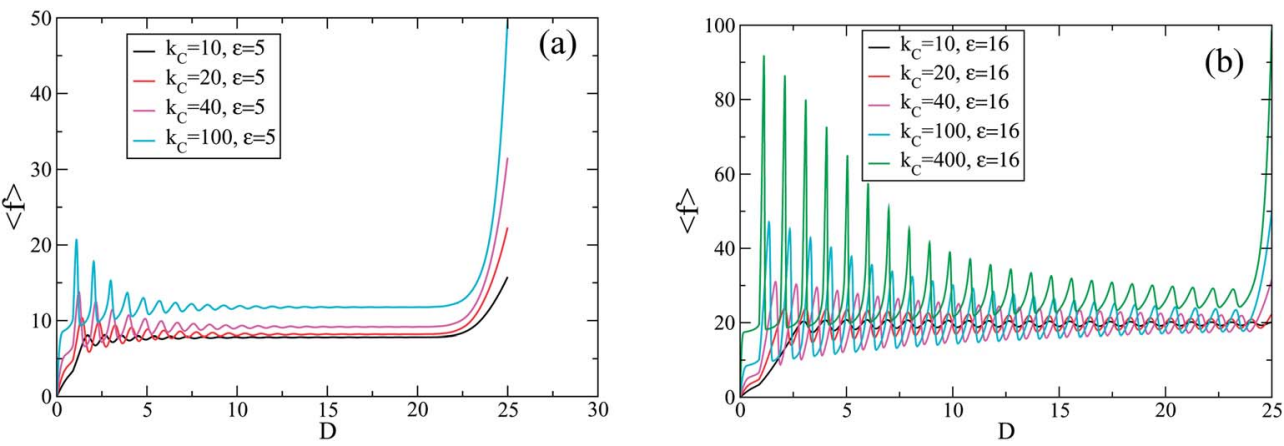

Fig. 2 The equilibrium force-displacement diagrams calculated according to eqn (11). The sawtooth structure becomes more pronounced with increasing adsorption energy $\varepsilon$ and spring constant $k_{\mathrm{c}}$.

corresponding thermodynamic condition reads $\mathscr{\mathscr { F }}(n, R)=\mathscr{\mathscr { F }}(n+$ $1, R)$. This condition also leads to the spike amplitude law $f_{\text {amp }}$ $\propto \exp (\varepsilon / n),{ }^{17}$ i.e. the spike amplitude gradually decreases in the process of chain detachment (i.e., with growing $n-c f$. Fig. 2).

This structure is more pronounced at larger adsorption energy $\varepsilon$ and cantilever spring constant $k_{\mathrm{c}}$. Thus, while the force oscillates, its mean value remains nearly constant in a broad interval of distances $D$, exhibiting a kind of plateau. Complementary information (for fixed $k_{\mathrm{c}}$ at different values of $\varepsilon$ ) is given in Fig. 3. One can verify that the plateau height is mainly determined by $\varepsilon$ whereas the spike amplitude is dictated by the cantilever spring constant $k_{\mathrm{c}}$.

It should be noted that similar spikes have been indeed observed in experiments on single-molecule stretching of proteins due to unfolding of the multidomain biopolymer structure. ${ }^{21}$ As far as the size of each such domain is much larger than that of a single segment, resolving this "sawtooth" behavior is significantly easier than in the present case of monomer peeling.

\subsection{Strong polymer-substrate friction}

In this limit one has to take into account the specific geometry of an AFM experiment, shown in Fig. 1 (right panel). For simplicity, an infinite friction of the polymer at the surface is assumed. The adsorbed polymer portion may be considered as a two-dimensional self-avoiding chain comprising $N-n$ segments. The last contact point (marked as $c$ in Fig. 1) can move due to adsorption or desorption elementary events. In ref. 32 this was classified as the sticky case. In Fig. $1, D$ is the distance from the cantilever base to the substrate, $R_{z}$ is the height of the cantilever tip above the substrate, and $R$ is the distance between the cantilever tip and the contact point $c$. Eventually, $R_{x}$ is the lateral distance between the cantilever base and the contact point $c$. One may assume that initially the desorbed portion of $n$ segments has occupied a distance of $R_{x}$

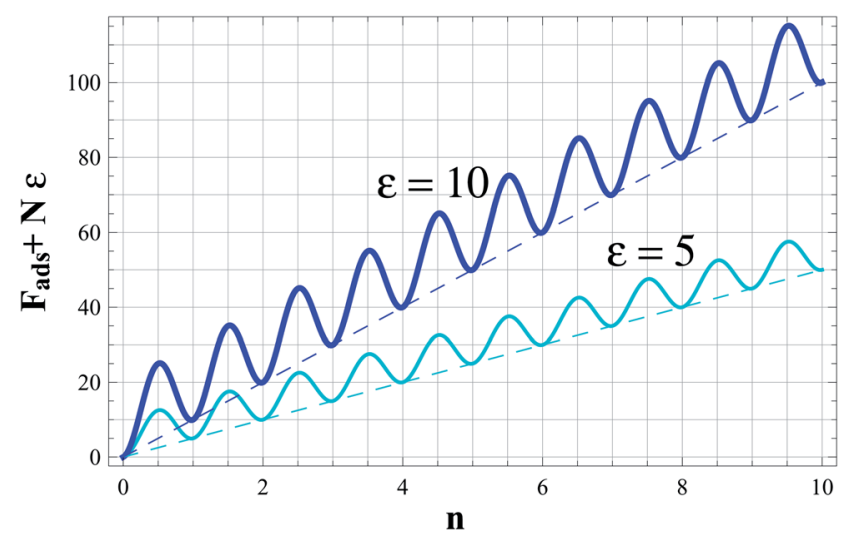

Fig. 4 Adsorption-desorption potential profiles for two different strengths $\varepsilon=5$ and $\varepsilon=10$ of surface attraction. The integer $n$-values correspond to minima whereas at half-integer $n$-values the potential has local maxima. The dashed lines denote the corresponding contact potential $F_{\text {ads }}=-\varepsilon(N-n)$ which has been used in Section 2 .
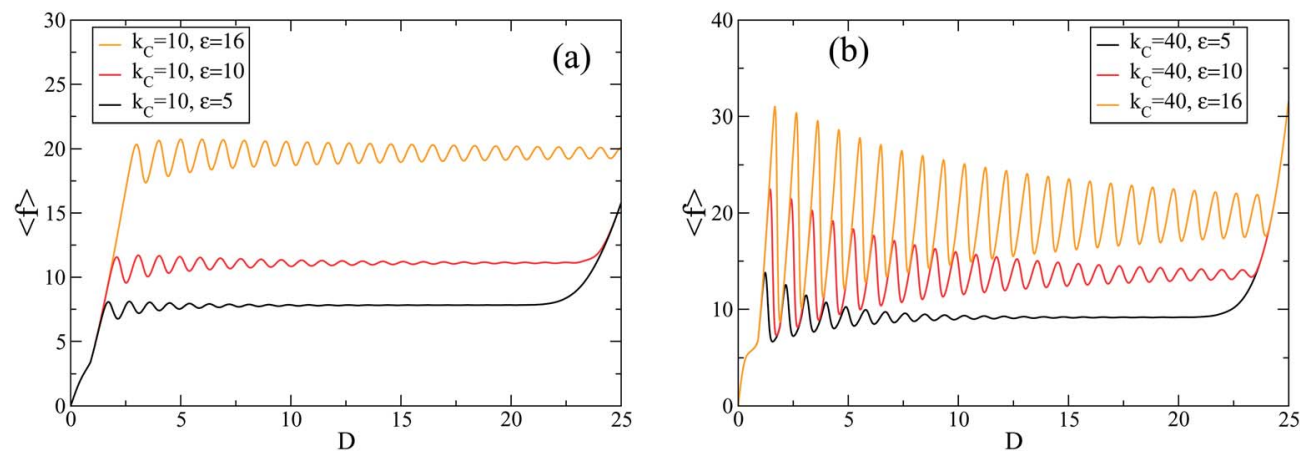

Fig. 3 The equilibrium force-displacement diagrams calculated according to eqn (11). The same as in Fig. 2 but for fixed $k_{c}$ and different $\varepsilon$. 
which, due to self-avoiding 2D-configurations of an adsorbed chain, equals $R_{x}^{2} \approx b^{2} n^{2 \nu}$ (where $\nu=3 / 4$ ). Therefore, in fact we assume that a concrete realization of adsorbed chain conformation is at best represented by the most probable one.

The specific geometry of the AFM experiment in the case of strong polymer-substrate friction (shown in Fig. 1) brings about changes only in the cantilever partition function, i.e., instead of eqn (5), one has

$$
\begin{aligned}
\Xi_{\mathrm{can}}(D, R) & =\exp \left[-\frac{k_{\mathrm{c}}}{2 k_{\mathrm{B}} T}\left(D-R_{z}\right)^{2}\right] \\
& =\exp \left[-\frac{k_{\mathrm{c}}}{2 k_{\mathrm{B}} T}\left(D-\sqrt{R^{2}-b^{2} n^{2 v}}\right)^{2}\right]
\end{aligned}
$$

As a result, the total partition function in this case is given by

$$
\begin{aligned}
\Xi_{\mathrm{tot}}(D)= & \sum_{n=0}^{N} \exp [\varepsilon(N-n)] \\
& \times \int_{b n^{\nu}}^{b n} \mathrm{~d} R \exp [n \mathscr{G}(\tilde{f})] \exp \left[-\frac{k_{\mathrm{c}}}{2 k_{\mathrm{B}} T}\left(D-\sqrt{R^{2}-b^{2} n^{2 v}}\right)^{2}\right] \\
& \times \theta\left(D-\sqrt{R^{2}-b^{2} n^{2 v}}\right)
\end{aligned}
$$

where again the variable $\tilde{f}$ should be excluded in favor of $R / b n$ by means of the relationship $\tilde{f}=\mathscr{L}^{-1}(R / b n)$. In eqn (13) the following constraints

$$
\begin{aligned}
& b n^{v}<R<b n, \\
& R_{z}=\sqrt{R^{2}-b^{2} n^{2 v}}<D,
\end{aligned}
$$

have been taken into account.

The corresponding free energy functional in terms of dynamical variables $n$ and $R$ has the following form

$$
\mathscr{F}(n, R)=-k_{\mathrm{B}} T \varepsilon(N-n)-k_{\mathrm{B}} T n \mathscr{G}(\tilde{f})+\frac{k_{\mathrm{c}}}{2}\left(D-\sqrt{R^{2}-b^{2} n^{2 v}}\right)^{2} .
$$

The average force, which is measured in AFM experiments, is given by

$$
\begin{aligned}
\left\langle f_{z}\right\rangle= & \frac{k_{\mathrm{c}}}{\Xi_{\mathrm{tot}}(D)} \sum_{n=0}^{N} \int_{b n^{\nu}}^{b n} \mathrm{~d} R\left(D-\sqrt{R^{2}-b^{2} n^{2 v}}\right) \\
& \times \exp \left[\varepsilon(N-n)+n \mathscr{S}(\tilde{f})-\frac{k_{\mathrm{c}}}{2 k_{\mathrm{B}} T}\left(D-\sqrt{R^{2}-b^{2} n^{2 v}}\right)^{2}\right] \\
& \times \theta\left(D-\sqrt{R^{2}-b^{2} n^{2 v}}\right) .
\end{aligned}
$$

\section{Dynamics of desorption}

In our recent paper ${ }^{33}$ we have studied a single polymer forceinduced desorption kinetics by making use of the notion of tensile blobs as well as by means of Monte Carlo and molecular dynamics simulations. It was clearly demonstrated that the total desorption time $\left\langle\tau_{\mathrm{d}}\right\rangle$ scales with polymer length $N$ as $\left\langle\tau_{\mathrm{d}}\right\rangle \propto N^{2}$.

In order to treat a realistic AFM experiment in which the cantilever-substrate distance changes with constant velocity $v_{\mathrm{c}}$, i.e., $D(t)=D_{0}+v_{\mathrm{c}} t$, one has to consider the AFM tip dynamics. With this in mind, we will develop a coarsegrained stochastic model based on the free-energy functional eqn (8). Before proceeding any further, we need to define the adsorption-desorption potential profile $F_{\text {ads }}(n)$. This plays the role of the potential of mean force (PMF) which depends on $n$.

\subsection{Stochastic model}

In the Helmholtz free-energy functional $\mathscr{F}(n, R)$, given by eqn (8) and (15), the free energy of the adsorbed portion is given by a simple contact potential, $F_{\text {ads }}=-k_{\mathrm{B}} T \varepsilon(N-n)$, where $n$ is an integer number in the range $0 \leq n \leq N$. Considering desorption dynamics (see below), we will treat $n$ as a continuous variable with a corresponding adsorption-desorption energy profile satisfying the following conditions:

1. For integer $n$-values the energy profile has minima whereby we use the contact potential $F_{\text {ads }}(n)=-k_{\mathrm{B}} T \varepsilon(N-n)$.

2 . For half-integer values of $n$ the adsorption potential goes over maxima.

3. The activation barrier for monomer desorption, $\Delta E^{+}=$ $F_{\text {ads }}(n+1 / 2)-F_{\text {ads }}(n)$, and the corresponding adsorption activation barrier, $\Delta E^{-}=F_{\text {ads }}(n+1 / 2)-F_{\text {ads }}(n+1)$, are proportional to the adsorption strength $\varepsilon$ of the substrate whereby $\Delta E^{+}>\Delta E^{-}$.

4. The adsorption-desorption energy profile satisfies the boundary conditions: $F_{\text {ads }}(0)=-k_{\mathrm{B}} T \varepsilon N$ (a fully adsorbed chain) and $F_{\text {ads }}(N)=0$ (an entirely detached chain).

One can show (see Appendix B for more details) that the following energy profile, given as ( $c f$. Fig. 4)

$$
F_{\text {ads }}(n)=T \varepsilon\{1+\cos [(2 n+1) \pi]+n\}-k_{\mathrm{B}} T \varepsilon N,
$$

meets the conditions (1)-(4).

The total Helmholtz free energy for the frictionless substrate model is given by

$$
\begin{aligned}
\mathscr{F}(n, R)= & k_{\mathrm{B}} T \varepsilon\{1+\cos [(2 n+1) \pi]+n\} \\
& -k_{\mathrm{B}} T \varepsilon N-k_{\mathrm{B}} T n \mathscr{G}(\tilde{f})+\frac{k_{\mathrm{c}}}{2}(D-R)^{2},
\end{aligned}
$$

whereas for the strong polymer-substrate friction model we have

$$
\begin{aligned}
\mathscr{F}(n, R)= & k_{\mathrm{B}} T \varepsilon\{1+\cos [(2 n+1) \pi]+n\}-k_{\mathrm{B}} T \varepsilon N-k_{\mathrm{B}} \operatorname{Tn} \mathscr{S}(\tilde{f}) \\
& +\frac{k_{\mathrm{c}}}{2}\left(D-\sqrt{R^{2}-b^{2} n^{2 v}}\right)^{2} .
\end{aligned}
$$

These Helmholtz free energy functions govern the dissipative process, which is described by the stochastic (Langevin) differential equations

$$
\begin{aligned}
& \frac{\partial n}{\partial t}=-\lambda_{n} \frac{\partial}{\partial n} \mathscr{F}(n, R)+\xi_{n}(t) \\
& \frac{\partial R}{\partial t}=-\lambda_{R} \frac{\partial}{\partial R} \mathscr{F}(n, R)+\xi_{R}(t)
\end{aligned}
$$

where $\lambda_{n}$ and $\lambda_{R}$ are the Onsager coefficients. The random forces $\xi_{n}(t)$ and $\xi_{R}(t)$ describe Gaussian noise with means and correlators given by 


$$
\begin{aligned}
& \left\langle\xi_{n}(t)\right\rangle=\left\langle\xi_{R}(t)\right\rangle=0 \\
& \left\langle\xi_{n}(t) \xi_{n}(0)\right\rangle=2 \lambda_{n} k_{\mathrm{B}} T \delta(t) \\
& \left\langle\xi_{R}(t) \xi_{R}(0)\right\rangle=2 \lambda_{R} k_{\mathrm{B}} T \delta(t)
\end{aligned}
$$

Eqn (20) are usually referred to as the Onsager equations. ${ }^{26}$

The set of stochastic differential equations, eqn (20), can be treated by a time integration scheme. Each realization $(l)$ of the solution provides a time evolution of $n^{(l)}(t)$ and $R^{(l)}(t)$. In order to obtain mean values of the observables, these trajectories should be averaged over many independent runs $l=1,2, \square, \mathscr{N}$. For example, in order to obtain the average force, eqn (16), one should average over the runs

$$
\left\langle f_{z}(t)\right\rangle=\frac{k_{\mathrm{c}}}{\mathscr{N}} \sum_{l=1}^{\mathscr{N}}\left(D(t)-\sqrt{\left[R^{(l)}(t)\right]^{2}-b^{2}\left[n^{(l)}(t)\right]^{2 \nu}}\right)
$$

\subsection{Thermodynamic forces}

The thermodynamic forces which arise in eqn (20), i.e., $f_{n} \stackrel{\text { def }}{=}-\frac{\partial \mathscr{F}(n, R)}{\partial n}$ and $f_{R} \stackrel{\text { def }}{=}-\frac{\partial \mathscr{F}(n, R)}{\partial R}$, could be calculated explicitly for both frictionless and strong friction substrate models (see Appendix C). In the first case the force $f_{n}$ reads

$$
f_{n}=-k_{\mathrm{B}} T \varepsilon\{1-2 \pi \sin [(2 n+1) \pi]\}+k_{\mathrm{B}} T \frac{R \tilde{f}}{b n}+k_{\mathrm{B}} T \mathscr{G}(\tilde{f}) .
$$

For the strong friction case, eqn (19) leads to a more complicated expression for the thermodynamic force:

$$
\begin{aligned}
f_{n}= & -k_{\mathrm{B}} T \varepsilon\{1-2 \pi \sin [(2 n+1) \pi]\}+k_{\mathrm{B}} T \frac{R \tilde{f}}{b n}+k_{\mathrm{B}} T \mathscr{G}(\tilde{f}) \\
& +\nu b^{2} k_{\mathrm{c}}\left(\frac{D}{\sqrt{R^{2}-b^{2} n^{2 v}}}-1\right) .
\end{aligned}
$$

The force $f_{R}$ for the frictionless substrate model is given by (see Appendix C for more details):

$$
f_{R}=-\frac{k_{\mathrm{B}} T \tilde{f}}{b}+k_{\mathrm{c}}(D-R) .
$$

For the model, given by eqn (19), the corresponding force reads

$$
f_{R}=-\frac{k_{\mathrm{B}} T \tilde{f}}{b}+k_{\mathrm{c}} R\left(\frac{D}{\sqrt{R^{2}-b^{2} n^{2 \nu}}}-1\right) .
$$

Finally, the variable $\tilde{f}$ should be expressed in terms of $R / b n$ by making use of the relationship $\tilde{f}=\mathscr{L}^{-1}(R / b n)$, where $\mathscr{L}^{-1}(x)$ is the inverse Langevin function. A very good approximation for the inverse Langevin function, published in ref. 34, is given by

$$
\tilde{f}=\mathscr{L}^{-1}(R / b n) \approx \frac{R}{b n}\left[\frac{3-\left(\frac{R}{b n}\right)^{2}}{1-\left(\frac{R}{b n}\right)^{2}}\right]
$$

\subsection{Quasistationary approximation}

It could be shown that for a strongly stretched desorbed portion of the polymer chain, the $R$ variable rapidly relaxes to its quasi- stationary value (see Appendix D). In other words, $R$ can quickly adjust to the slow evolution of $n$ (governed by the Kramers process). In this quasi-stationary approximation $f_{R}=0$, and from eqn (25) one has $k_{\mathrm{c}}(D-R)=k_{\mathrm{B}} T \tilde{f} / b$, so that the following nonlinear equation for $R$ emerges

$$
k_{\mathrm{c}}[D(t)-R]=\frac{k_{\mathrm{B}} T}{b} \mathscr{D}^{-1}\left(\frac{R}{n b}\right)
$$

This could be represented as

$$
G(n, R ; t) \stackrel{\text { def }}{=} \frac{R}{b n}-\mathscr{L}\left(\frac{k_{\mathrm{c}} b[D(t)-R]}{k_{\mathrm{B}} T}\right)=0,
$$

i.e., the height $R$ is instantaneously coupled to the number of desorbed beads, $n$. Inserting eqn (27) into eqn (28), one obtains

$$
P(n, R ; t) \stackrel{\text { def }}{=} \frac{k_{\mathrm{c}} b[D(t)-R]}{k_{\mathrm{B}} T}-\left(\frac{R}{b n}\right)\left[\frac{3-\left(\frac{R}{b n}\right)^{2}}{1-\left(\frac{R}{b n}\right)^{2}}\right]=0 .
$$

The Onsager equation for the slow variable $n$ is given as

$$
\begin{aligned}
\frac{\partial n}{\partial t}= & \lambda_{n} f_{n}(n, R)+\xi_{n}(t) \\
= & \lambda_{n}\left[-k_{\mathrm{B}} T \varepsilon\{1-2 \pi \sin [(2 n+1) \pi]\}+k_{\mathrm{B}} T \frac{R \tilde{f}}{b n}+k_{\mathrm{B}} T \mathscr{G}(\tilde{f})\right] \\
& +\xi_{n}(t)
\end{aligned}
$$

where $\tilde{f}$ is determined by eqn (27).

Eventually, we get a system of so-called semi-explicit differential-algebraic equations (DAE) ${ }^{35}$

$$
\begin{aligned}
& \frac{\partial n}{\partial t}=\lambda_{n} f_{n}(n, R)+\xi_{n}(t) \\
& 0=G(n, R ; t)
\end{aligned}
$$

In this particular form of DAE one can distinguish between the differential variable $n(t)$ and the algebraic variable $R(t)$. Eqn (32) can be solved numerically by making use of an appropriate Runge-Kutta (RK) algorithm, as shown in Appendix E.

\subsection{Results}

We have solved numerically our stochastic model, given by eqn (31) and (29), for the case of the frictionless substrate. To this end we used the second order Runge-Kutta (RK) algorithm for stochastic differential-algebraic equations (see Appendix E for more details). The advantage of the stochastic differential equation approach as compared to the master equation method $^{23}$ is that the former one gives a more detailed (not averaged) dynamic information corresponding to each individual force-displacement trajectory (as is often in an experiment). The result of averaging over 300 runs is shown in Fig. 5 (left).

Fig. 5 (right panel) shows the resulting force-displacement diagram for $\varepsilon=5$ and different detachment velocities. It is worth noting that the "sawtooth" pattern can be seen for all investigated detachment velocities ranging between $v_{\mathrm{c}}=$ 

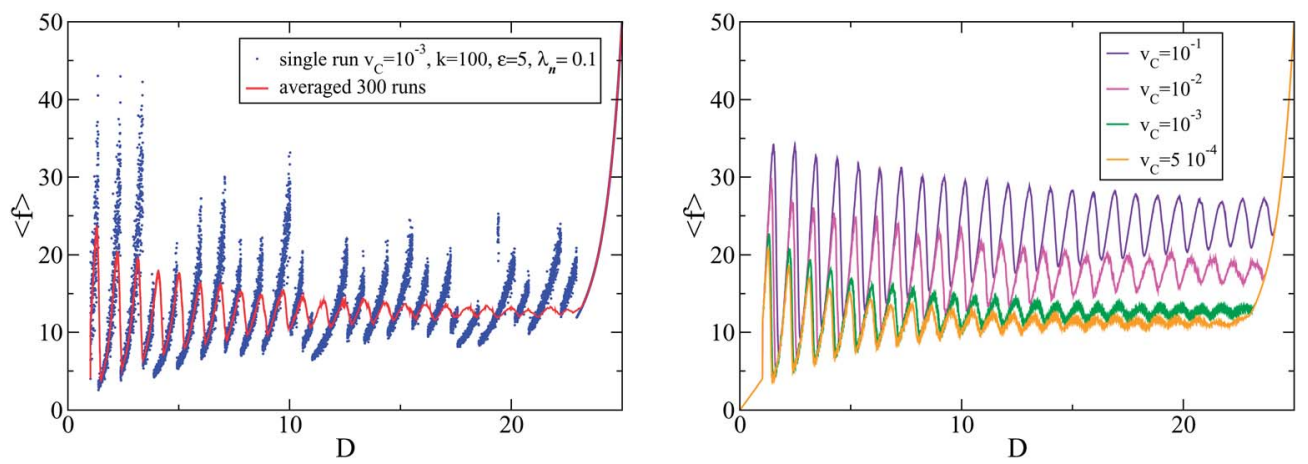

Fig. 5 (left panel) The effect of averaging over many desorption events. The blue line shows a single run, whereas the red line demonstrates the result of averaging over 300 runs. The detachment velocity $v_{c}=100$, the cantilever spring $k_{\mathrm{c}}=100$, adsorption energy $\varepsilon=5$, and the Onsager coefficient $\lambda=0.1$. (right panel) The dynamic force-displacement diagram for $\varepsilon=5$ and different detachment velocities $v_{\mathrm{c}}$ (see legend).

$5 \times 10^{-4}$ and $v_{\mathrm{c}}=10^{-2}$. For larger velocities the plateau height of the force increases substantially. In other words, the mean detachment force increases as the AFM-tip velocity increases and the bond stretching between successive monomers becomes stronger.

We have also studied the detachment force behavior as well as the cantilever tip distance from the substrate at the moment of a full detachment (i.e. when $n=N$ ), by repeating the detachment procedure $10^{4}$ times and plotting the probability distribution functions (PDF) for different adsorption energies $\varepsilon$ and detachment velocities $v_{\mathrm{c}}-$ Fig. 6 . As one can see from Fig. 6a and $\mathrm{b}$, both the average and the dispersion of detachment force grow with $v_{\mathrm{c}}$ which agrees with findings for reversible (i.e., when a broken bond can rebind) bond-breaking dynamics. ${ }^{36}$ In contrast, the mean cantilever tip distance $R$ variance decreases and its average value increases with growing $v_{\mathrm{c}}(c f$. Fig. 6c and d).

The average detachment force dependence on cantilever velocity $v_{\mathrm{c}}$ is a widely covered subject in the literature in the context of biopolymer unfolding ${ }^{37-39}$ or forced separation of two adhesive surfaces. ${ }^{36,40,41}$ Fig. $7 \mathrm{a}$, which shows the results of our calculations, shows the characteristic features discussed also in ref. 40. One observes a well expressed crossover from a shallowslope for relatively small detachment rates to a steep-slope
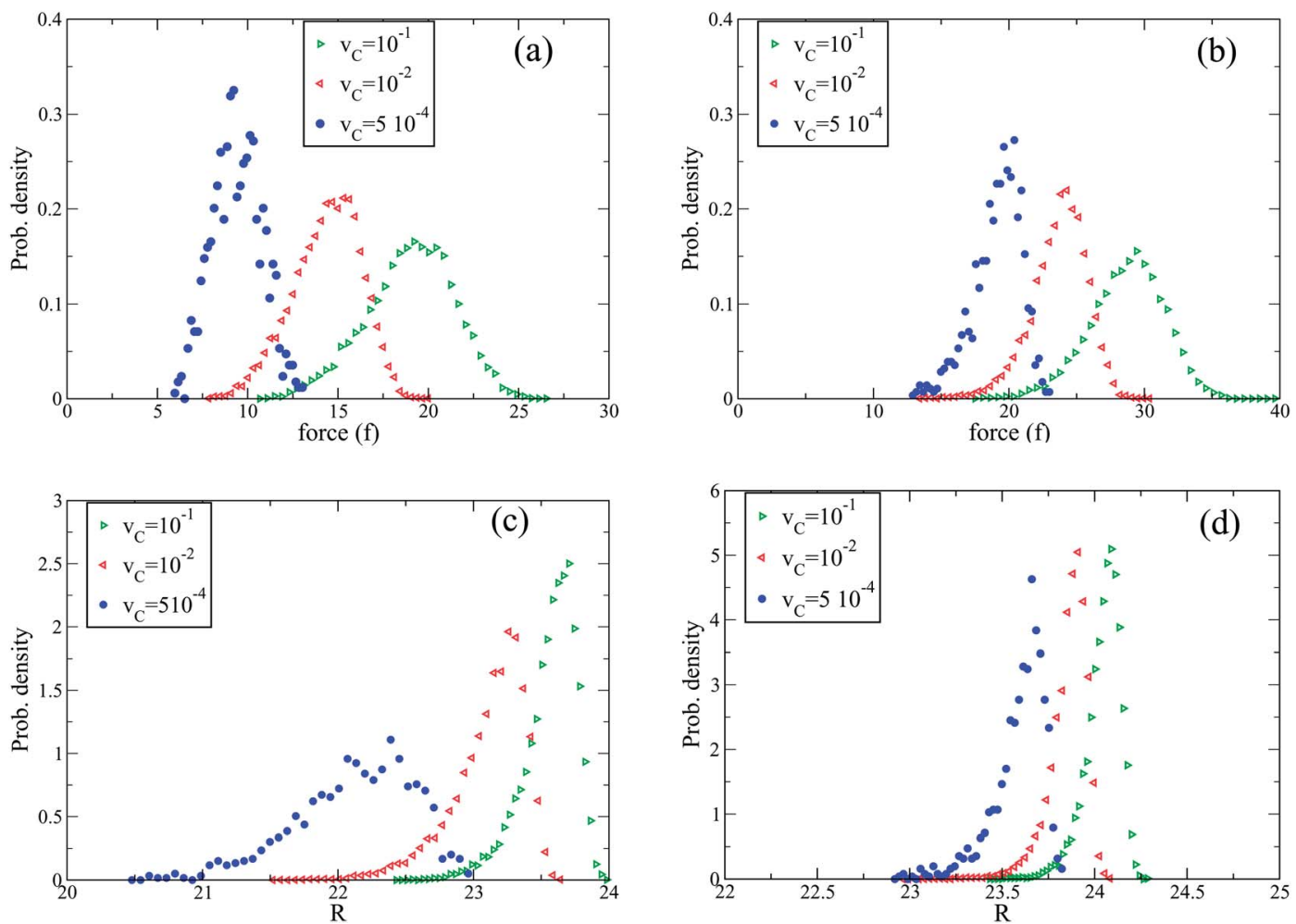

Fig. 6 The normalized PDF for detachment forces at $\varepsilon=5$ (a), and $\varepsilon=8$ (b), as well as for different detachment velocities $v_{c}$ (shown in the legends). The PDFs for the detachment distance $R$ of the cantilever tip at $\varepsilon=5$ (c) and $\varepsilon=8$ (d). 

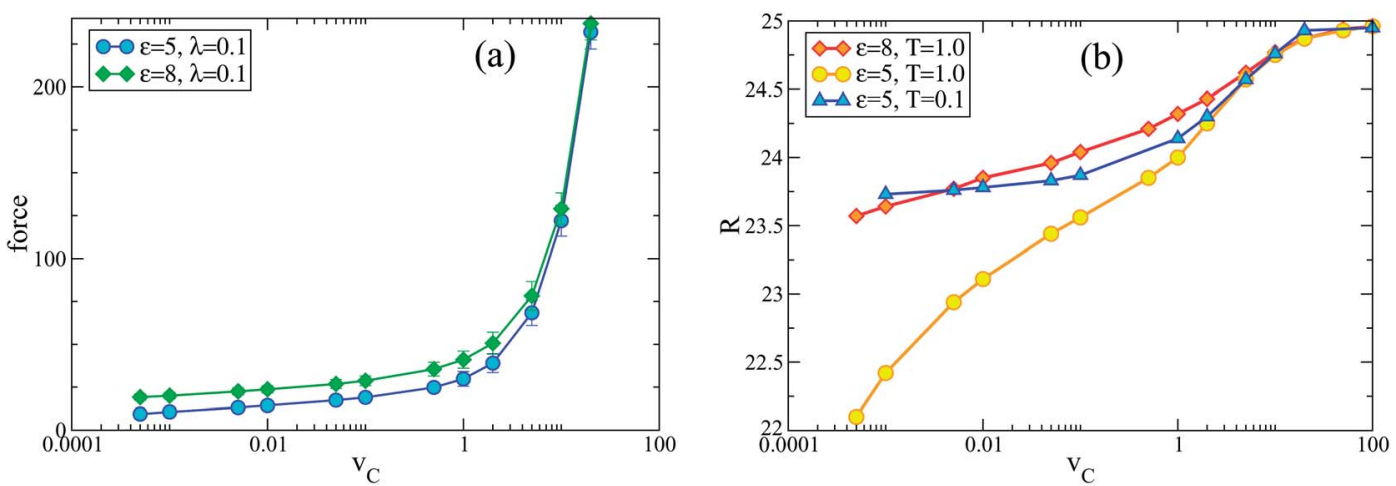

Fig. 7 The average detachment force (a), and cantilever tip distance (b), vs. detachment velocity $v_{\mathrm{c}}$ for two different adsorption energies $\varepsilon=5$ and $\varepsilon=8$. In addition, the distance $R$ is plotted in (b) for two different temperatures, $T=1.0$ and $T=0.1$, both at the same adsorption strength $\varepsilon=$ 5.0. Evidently, with growing $T$ the elasticity of the coil decreases, the coil itself becomes stiffer and therefore detaches from the substrate at lower height $R$. This entropic effect is well expressed at sufficiently low pulling velocity only.

region as the detachment speed increases. One remarkable feature is that this crossover practically does not depend on the adsorption energy $\varepsilon$ : the curve is merely shifted upwards upon increasing $\varepsilon$. Therefore, the crossover is not related to a competition between the Kramers rate and the cantilever velocity but rather accounts for the highly nonlinear chain stretching as the velocity $v_{\mathrm{c}}$ increases. The corresponding detachment distance of the cantilever tip $R$ (detachment height), Fig. 7b, reveals a specific sigmoidal shape in agreement with the results based on the master equation. ${ }^{23}$ At low velocities of pulling, $v_{c}$, when the chain still largely succeeds in relaxing back to equilibrium during detachment, an interesting entropy effect is manifested in Fig. 7b: the (effectively) stiffer coil at $T=1.0$ leaves the substrate at lower values of $R$ than in the case of the colder system, $T=0.1$. As the pulling velocity increases, however, this entropic effect vanishes and the departure from the substrate is largely governed by the stretching of the bonds rather than of the coil itself whereby the difference in behavior between $T=1.0$ and $T=0.1$ disappears.

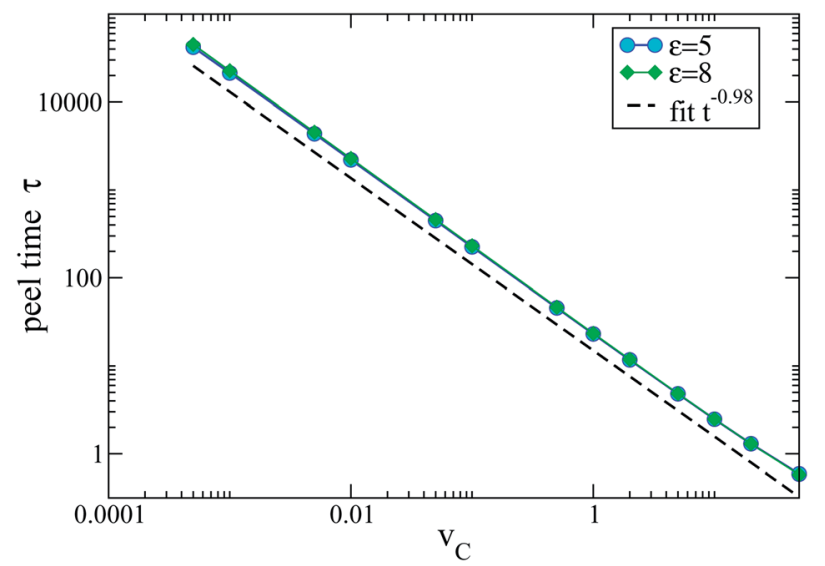

Fig. 8 The average detachment (peeling) time $\tau_{\text {det }}$ versus detachment velocity $v_{\mathrm{c}}$ for two different adsorption energies $\varepsilon=10$ and $\varepsilon=16$. The inversely proportional dependence, $\tau_{\text {det }} \sim 1 / v_{c}$, agrees well with previous theoretical findings. ${ }^{41}$
Eventually, as can be seen from Fig. 8, the total detachment (peel) time $\tau_{\text {det }} v s$. velocity $v_{\mathrm{c}}$ relationship has a well-defined power-law behavior, $\tau_{\mathrm{det}} \sim 1 / v_{\mathrm{c}}{ }^{\alpha}$, with the power $\alpha \approx 1$, in line with previous theoretical findings. ${ }^{41}$

\section{MD simulations}

\subsection{The model}

In our MD simulations we use a coarse-grained model of a polymer chain of $N$ beads connected by finitely extendable elastic bonds. The bonded interactions in the chain are described by the frequently used Kremer-Grest potential, $V^{\mathrm{KG}}(r)$ $=V^{\mathrm{FENE}}(r)+V^{\mathrm{WCA}}(r)$. The FENE (finitely extensible nonlinear elastic) potential is given by

$$
V^{\mathrm{FENE}}=-\frac{1}{2} k r_{0}^{2} \ln \left[1-\left(\frac{r}{r_{0}}\right)^{2}\right]
$$

with $k=30 \varepsilon / \sigma^{2}$ and $r_{0}=1.5 \sigma$.

In order to allow properly for excluded volume interactions between bonded monomers, the repulsion term is taken as the Weeks-Chandler-Anderson (WCA) potential (i.e., the shifted and truncated repulsive branch of the Lennard-Jones potential) given by

$$
V^{\mathrm{WCA}}(r)=4 \varepsilon_{\mathrm{LJ}}\left[(\sigma / r)^{12}-(\sigma / r)^{6}+1 / 4\right] \theta\left(2^{1 / 6} \sigma-r\right)
$$

with $\theta(x)=0$ or 1 for $x<0$, or $x \geq 0$, and $\varepsilon_{\mathrm{LJ}}=1, \sigma=1$. The overall potential $V^{\mathrm{KG}}(r)$ has a minimum at bond length $r_{\text {bond }} \approx$ 0.96. The nonbonded interaction between monomers are taken into account by means of the WCA potential, eqn (34). Thus, the interactions in our model correspond to good solvent conditions.

The substrate in the present investigation is considered simply as a structureless adsorbing plane, with a Lennard-Jones potential acting with strength $\varepsilon_{\mathrm{s}}$ in the perpendicular $z$-direction, $V^{\mathrm{LJ}}(z)=4 \varepsilon_{\mathrm{s}}\left[(\sigma / z)^{12}-(\sigma / z)^{6}\right]$. In our simulations we consider as a rule the case of strong adsorption $\varepsilon_{\mathrm{s}} / k_{\mathrm{B}} T=5-20$, where $k_{\mathrm{B}} T$ is a temperature of the Langevin thermal bath described below. 
The dynamics of the chain is obtained by solving the Langevin equations of motion for the position $\mathbf{r}_{n}=\left[x_{n}, y_{n}, z_{n}\right]$ of each bead in the chain,

$$
m \ddot{\mathbf{r}}_{n}=\mathbf{F}_{n}^{j}+\mathbf{F}_{n}{ }^{\mathrm{WCA}}-\gamma \dot{\mathbf{r}}_{n}+\mathbf{R}_{n}(t)(1, \ldots, N)
$$

which describes the Brownian motion of a set of bonded particles.

The influence of solvent is split into slowly evolving viscous force and rapidly fluctuating stochastic force. The random Gaussian force $\mathbf{R}_{n}$ is related to friction coefficient $\gamma=0.25 \tau^{-1}$ by the fluctuation-dissipation theorem. The integration step is $\tau=0.005$ and time is measured in units of $\sqrt{m \sigma^{2} / \varepsilon_{\mathrm{LJ}}}$, where $m$ denotes the mass of the polymer beads, $m=1$. In all our simulations the velocity-Verlet algorithm was used to integrate equations of motion (35).

The molecule is pulled by a cantilever at constant velocity $\mathbf{V}$ $=\left[0,0, v_{\mathrm{c}}\right]$. The cantilever is imitated by two beads connected by a harmonic spring and attached to one of the ends of the chain. $\dagger$

The mass of beads $m_{\mathrm{c}}$, forming the cantilever, was set either to $m_{\mathrm{c}}=1$ or to 25 . The equilibrium size of this harmonic spring was set to 0 and the spring constant was varied in the range $k_{\mathrm{c}}=$ $50-400 \varepsilon_{\mathrm{LJ}} / \sigma^{2}$. The hydrodynamic radius $a$ of beads composing the cantilever was varied by changing the friction coefficient $\gamma_{\mathrm{c}}$ $=0.25-25 \tau^{-1}$, taking into account the Stokes' law, $\gamma_{c}=6 \pi \eta a$, where $\eta$ is the solvent viscosity.

Taking the value of the thermal energy $k_{\mathrm{B}} T \approx 4.11 \times 10^{-21} \mathrm{~J}$ at $k_{\mathrm{B}} T=300 \mathrm{~K}$, the typical Kuhn length of $\sigma=1 \mathrm{~nm}$ and the mass of the coarse-grained monomer as $m \approx 10^{-25} \mathrm{~kg}$ sets the unit of time in our simulations which is given in $10^{-12} \mathrm{~s}=1 \mathrm{ps}$. The velocities used in simulations are in units of $10^{-4} \div 10^{-1}$ $\mathrm{nm} \mathrm{ps}{ }^{-1} \approx 10^{-1} \div 10^{2} \mathrm{~m} \mathrm{~s}^{-1}$. Spring constants of our cantilever in real units are: $k_{\mathrm{c}}=50 \div 400 k_{\mathrm{B}} T \mathrm{~nm}^{-2}=0.2 \div 1.6 \mathrm{~N} \mathrm{~m}^{-1}$.

Two typical snapshots of a polymer chain during slow detachment from an adsorbing substrate with different strengths of adsorption, $\varepsilon_{\mathrm{s}}=2.5$ and $\varepsilon_{\mathrm{s}}=20$ are shown in Fig. 9 . Evidently, the chain is much more stretched for the strongly attractive substrate where all adsorbed monomers stick firmly to the surface.

\subsection{MD results}

As we have already seen in Section 3.4, the averaging of the force profile over many runs reveals the inherent sawtooth-structure of the force $v s$. distance dependence (see Fig. 5), which is otherwise overshaded by thermal noise. Our MD simulation results, depicted in Fig. 10, show the same tendency against the noisy background of a single detachment event. Therefore, for better clarity and physical insight, all our graphic results that are given below result from such an averaging procedure.

Fig. 11a shows how adsorption energy $\varepsilon_{\mathrm{s}}$ affects the force $f v s$. distance $D$ relationship. Apparently, with increasing $\varepsilon_{\mathrm{s}}$ the mean

$\dagger$ This setup is different from the one used by S. Iliafar et al. ${ }^{19}$ In their study a harmonic spring was connected to a "big" monomer (with large friction coefficient) on one side, and to a mobile wall on the other side. In our case the harmonic spring spans two beads.
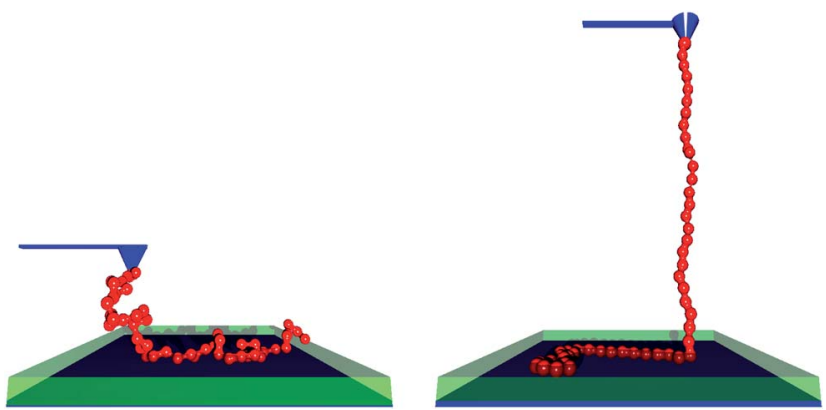

Fig. 9 Snapshots of a polymer chain with $N=50$ monomers during detachment from a substrate at $\varepsilon_{\mathrm{s}}=2.5$ (left) and $\varepsilon_{\mathrm{s}}=20$ (right). Here $v_{\mathrm{c}}$ $=0.0001$. The interaction range of the adsorption potential is shaded (transparent) green. The cantilever tip is shown schematically in blue. One may clearly see that the polymer chain is more relaxed (less stretched) at $\varepsilon_{\mathrm{s}}=2.5$, and the adsorbed monomers do not stick tightly to the surface but partially exit the range of surface adsorption.

force (plateau height) is found to increase in agreement with our equilibrium theory results, given in Fig. 3. As suggested by our recent theory, ${ }^{27,29}$ the plateau height goes up as $f_{\mathrm{p}} \propto \varepsilon_{\mathrm{s}}{ }^{1 / 2}$, or as $f_{\mathrm{p}} \propto \varepsilon_{\mathrm{s}}$, for relatively small or large $\varepsilon_{\mathrm{s}}$ values, respectively. The amplitude of spikes increases with growing $\varepsilon_{\mathrm{s}}$ too, in line with the equilibrium findings (see Fig. 3). Moreover, as found by Jagota et al., ${ }^{17}$ the amplitude of spikes follows an exponential law, $f_{\text {amp }} \propto \exp \left(\varepsilon_{\mathrm{s}} / n\right)$, where $n$ is the number of desorbed polymer segments. On the other hand, the comparison of Fig. 11b and 2 suggests that the stiffness of the cantilever spring constant $k_{\mathrm{c}}$ affects mainly the spike amplitude especially at large $\varepsilon_{\mathrm{s}}$.

Eventually, we demonstrate the impact of the cantilever velocity, $v_{\mathrm{c}}$, as well as of its mass, $m_{\mathrm{c}}$, and friction coefficient, $\gamma_{\mathrm{c}}$, on the force-distance profile. Apparently, these parameters affect to different extents the observed force-distance relationship. Similar to the results, obtained for our coarse-grained

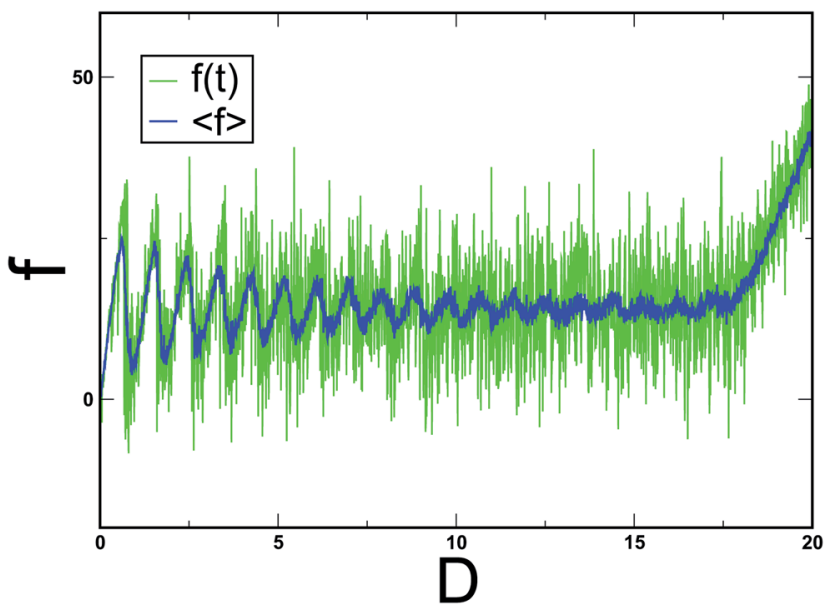

Fig. 10 Comparison of averaged force measured at the cantilever vs. single realization of the desorption experiment. Force $f$ at the cantilever was calculated by monitoring extensions $\Delta z_{c}$ of a harmonic spring, i.e. $f=k_{\mathrm{C}} \Delta z_{\mathrm{C}}$. Here $N=20, k_{\mathrm{C}}=50 \varepsilon_{\mathrm{LJ}} / \sigma^{2}, v_{\mathrm{C}}=10^{-4} \sigma / \tau$ and $\varepsilon_{\mathrm{s}} /$ $k_{\mathrm{B}} T=20 . m_{\mathrm{c}}=1$ and $\gamma_{\mathrm{c}}=0.25$. 

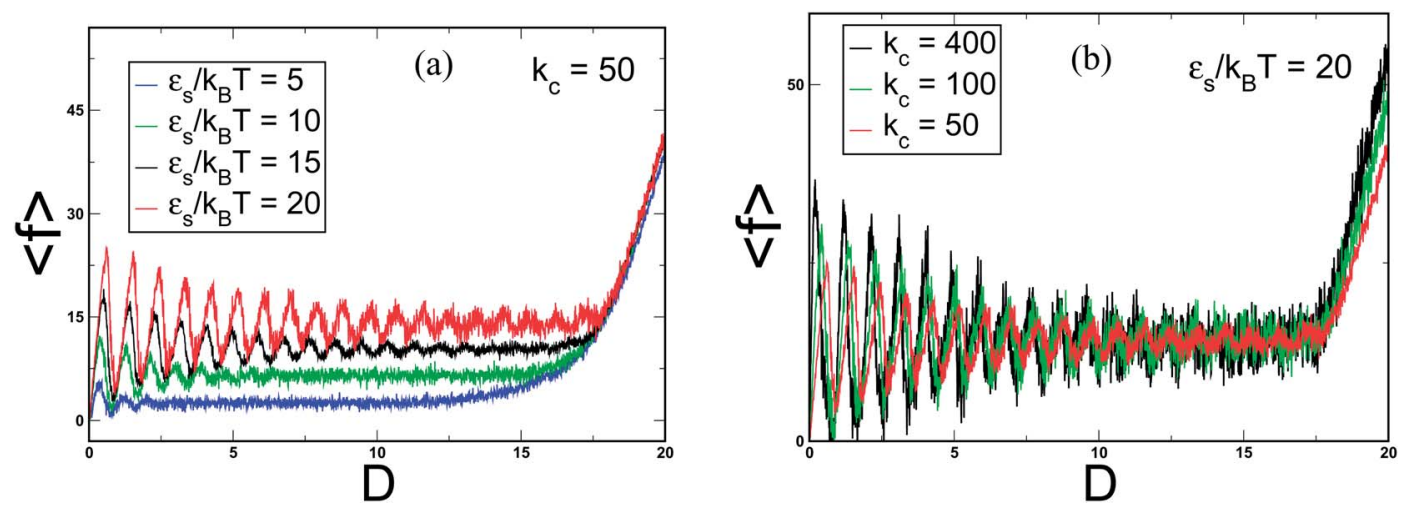

Fig. 11 Time-averaged force $\langle f\rangle$ at the cantilever as a function of a distance from the substrate $D$ during chain detachment. Here $N=20, v_{\mathrm{c}}=$ $10^{-4} \sigma / \tau, m_{\mathrm{c}}=1$ and $\gamma_{\mathrm{c}}=0.25$. Presented results are for different adsorption strengths $\varepsilon_{\mathrm{s}}$, as indicated in the legend, and $k_{\mathrm{c}}=50 \varepsilon_{\mathrm{LJ}} / \sigma^{2}$ (a). Figure (b) presents results for $\varepsilon_{\mathrm{s}}=20$ and different spring constants $k_{\mathrm{c}}$. Each curve originates from $10^{3}$ independent simulations.

model in Section 2, in the MD simulation data the plateau height increases less than twice upon the velocity increase of three orders of magnitude (see Fig. 12). Only for a very massive $\left(m_{\mathrm{c}}=25\right)$ and strong friction $\left(\gamma_{\mathrm{c}}=25\right)$, cantilever, the plateau height increases significantly and gains a slight positive slope (see Fig. 12d) whereby oscillations vanish. This occurs for the fastest detachment $v_{\mathrm{c}}=0.1 \sigma / \tau$. Evidently, this effect is related to the combined role of the friction force in the case of rapid detachment along with the much larger inertial force $\left(m_{\mathrm{c}}=25\right)$ whereby the substrate-induced oscillations are overshadowed by the increased effort of pulling. In contrast, neither Fig. 12b nor Fig. $12 \mathrm{c}$ indicates any major qualitative changes in the $f-v s$. $D$-behavior when medium-friction or mass cantilever alone is drastically changed.

The PDF of the detachment force and its velocity $v_{\mathrm{c}}$ dependence are shown in Fig. 13. Similar to that in Section 2, the average value and dispersion increase with increasing speed of pulling and this is weakly sensitive with regard to the
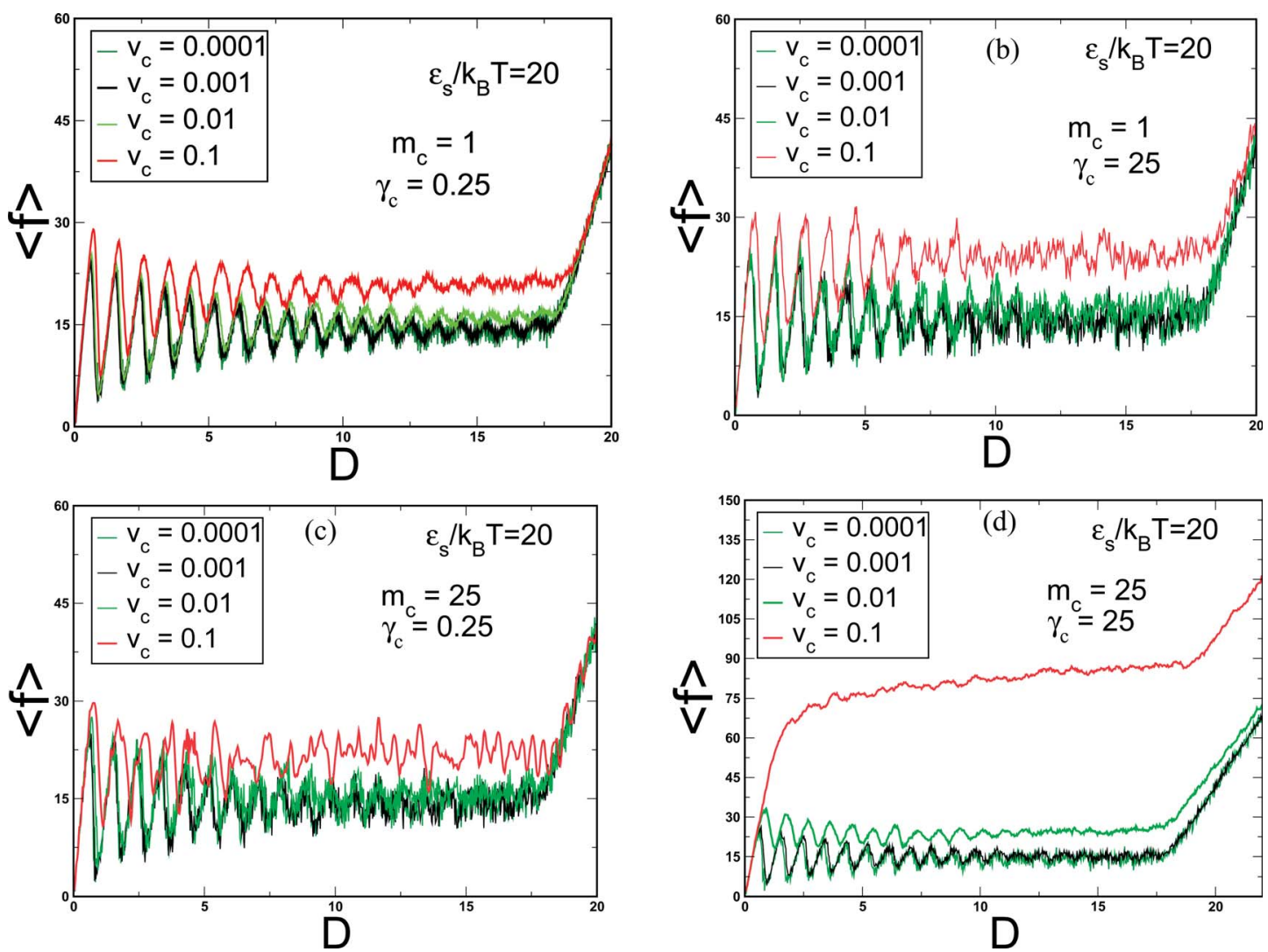

Fig. 12 Profiles of averaged force $\langle f\rangle v s$. distance above the substrate $D$ displayed for different pulling velocities $v_{\mathrm{C}}$. Here $N=20, k_{\mathrm{C}}=50 \varepsilon_{\mathrm{LJ}} / \sigma^{2}$ and $\varepsilon_{\mathrm{s}} / k_{\mathrm{B}} T=20$ and (a) $m_{\mathrm{c}}=1, \gamma_{\mathrm{c}}=1$, (b) $m_{\mathrm{c}}=1, \gamma_{\mathrm{c}}=25$, (c) $m_{\mathrm{c}}=25, \gamma_{\mathrm{c}}=0.25$, and (d) $m_{\mathrm{c}}=25, \gamma_{\mathrm{c}}=25$. 
adsorption strength of the substrate $\varepsilon_{\mathrm{s}}$. Remarkably, the mean detachment force $\left\langle f_{\mathrm{d}}\right\rangle$ shows a similar nonlinear dependence on $\ln v_{\mathrm{c}}(c f$. Fig. 7a). The crossover position does not change practically as the adhesion strength is varied, and the variation of the other parameters $\left(m_{\mathrm{c}}=1 \rightarrow 25, \gamma_{\mathrm{c}}=0.25 \rightarrow 25\right)$ towards a massive and strong-friction cantilever renders this crossover considerably more pronounced.

The complementary PDF for the detachment height $R$ is given in Fig. 14a together with the corresponding average $\langle R\rangle v s$. $v_{\mathrm{c}}$ relationship. As predicted by our analytical model, $c f$. Section 2 , the height of the final detachment of the chain from the substrate becomes larger for faster peeling $v_{\mathrm{c}}$ and stronger adhesion $\varepsilon$, which is consistent with the MD data. One can see again the typical sigmoidal-shape in the $\langle R\rangle v s . v_{\mathrm{c}}$ dependence.

The two panels for different temperatures, shown in Fig. 14b, indicate a smaller increase in $\langle R\rangle$ at the higher temperature, provided the pulling velocity $v_{\mathrm{c}}$ is sufficiently small too. This can be readily understood in terms of entropic (rubber) elasticity of polymers and represents a case of delicate interplay between entropy and energy-dominated behavior. It is well known that a polymer coil becomes less elastic (i.e., it contracts) upon a temperature increase, $c f$. the lowest (grey) curve in Fig. 14b, (left panel) at $T=1.0$, so that $R$ is smaller than in the corresponding lowest curve for $T=0.1$ in the right panel of Fig. 14b. This occurs at low values of $v_{\mathrm{c}}$. On the other hand, the softer chain (at $T=0.1$ ) stretches more easily and, therefore, $R$ goes up to $\approx 90$ for the highest speed $v_{\mathrm{c}}=10^{-1}$ instead of $R \approx 85$ for $T=1.0, v_{\mathrm{c}}$ $=10^{-1}$. This entropic effect is well expressed at weak attraction to the surface, $\varepsilon_{\mathrm{s}} / k_{\mathrm{B}} T=2.25$, which does not induce strong stretching of the bonds along the chain backbone. In contrast, at high $\varepsilon_{\mathrm{S}} / k_{\mathrm{B}} T=20$, the bonds extend so strongly that the chain turns almost into a string and entropy effects become negligible. The energy cost of stretching then dominates and leads to higher values of $R$ at the higher temperature (cf. upper most green symbols in Fig. 14b) since it is now the elasticity of the bonds between neighboring segments which governs the
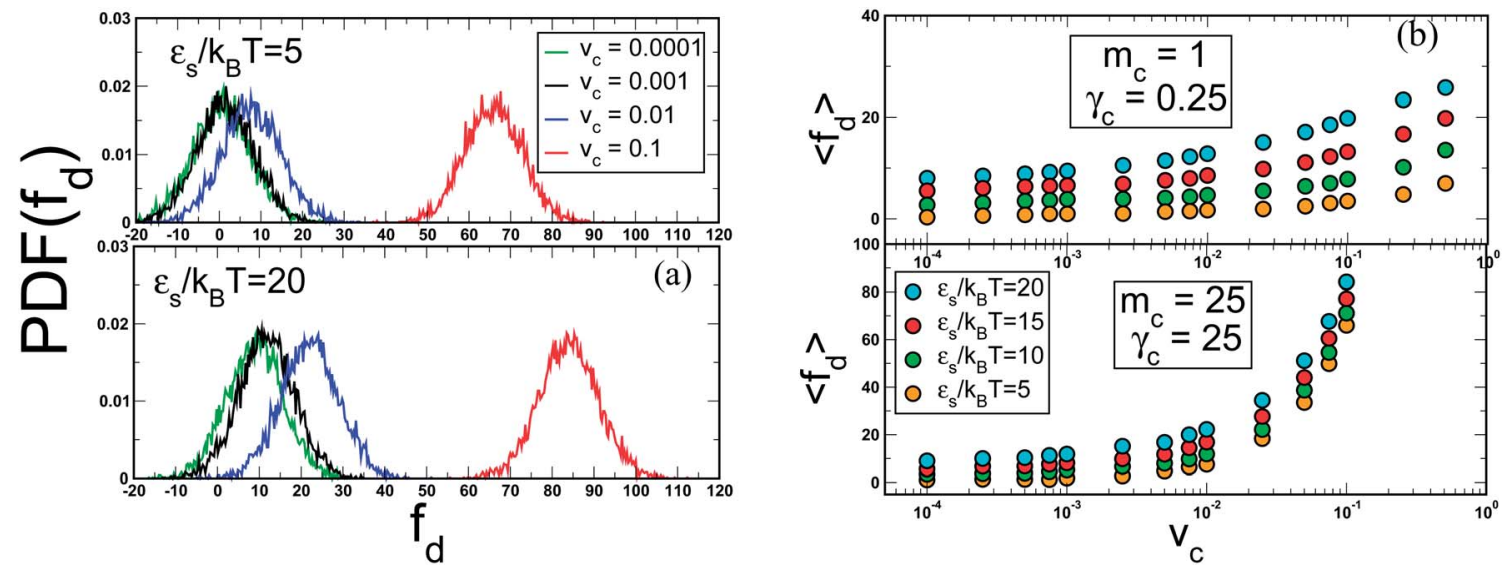

Fig. 13 (a) Probability distribution function of a force $f_{\mathrm{d}}$ at the cantilever measured at the moment of detachment (when the last monomer leaves the substrate). Here $N=20, k_{\mathrm{c}}=50 \varepsilon_{\mathrm{LJ}} / \sigma^{2}, \varepsilon_{\mathrm{s}} / k_{\mathrm{B}} T=20, m_{\mathrm{c}}=1$ and $\gamma_{\mathrm{c}}=0.25$. (b) Averaged force $\left\langle f_{\mathrm{d}}\right\rangle$ plotted versus pulling velocity $v_{\mathrm{c}}$ in the semilogaritmic scale. The values of parameters are the same.
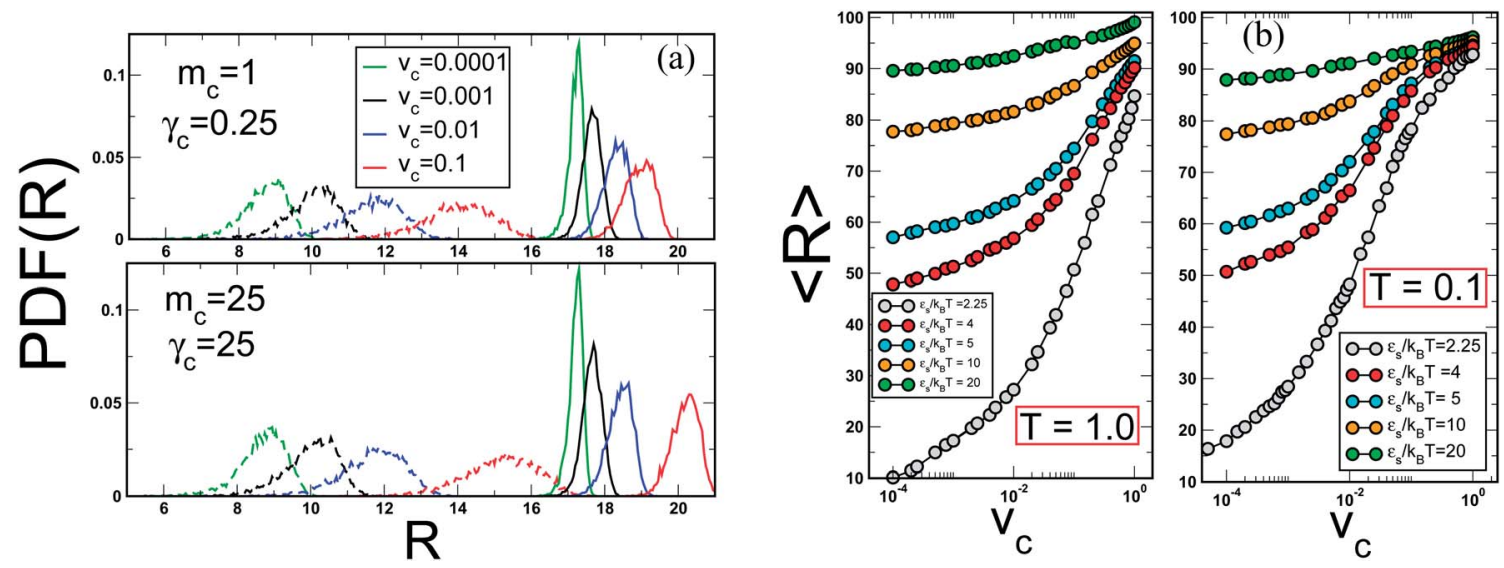

Fig. 14 (a) Probability distribution function of the detachment height $R$. The upper panel presents results for $m_{\mathrm{C}}=1$ and $\gamma_{\mathrm{c}}=0.25$ while the lower panel contains data for $m_{\mathrm{c}}=25$ and $\gamma_{\mathrm{c}}=25$. Solid lines represent results for $\varepsilon_{\mathrm{s}} / k_{\mathrm{B}} T=20$ while dashed lines represent results for $\varepsilon_{\mathrm{s}} / k_{\mathrm{B}} T=5$. Here $N$ $=20, k_{\mathrm{C}}=50 \varepsilon_{\mathrm{LJ}} / \sigma^{2}$. (b) Averaged detachment height $\langle R\rangle$ as a function of pulling velocity $v_{\mathrm{C}}$ in the semi-logarithmic scale plotted for different adsorption strengths as indicated: (left panel) $k_{\mathrm{B}} T=1.0$, (right panel) $k_{\mathrm{B}} T=0.1$. On both panels symbols plotted with the same color correspond to the same ratio of $\varepsilon_{\mathrm{S}} / k_{\mathrm{B}} T$ while the corresponding values of $\varepsilon_{\mathrm{s}}$ and $k_{\mathrm{B}} T$ differ. Here $N=100, k_{\mathrm{C}}=50 \varepsilon_{\mathrm{LJ}} / \sigma^{2}, m_{\mathrm{C}}=1$ and $\gamma_{\mathrm{C}}=0.25$. 
physics of detachment. In this case the elastic constant of the bonds effectively decreases with an increase of $T$ so that the distance of detachment $R$ in the left panel of Fig. 14b for $T=1.0$ is higher than that for $T=0.1$ in the right panel.

\section{Discussion}

We have demonstrated in this paper that a simple theory, based on the Onsager stochastic equations, yields an adequate description of a typical AFM experiment within the displacement-control mode. This approach makes it possible to relax most of the restrictions inherent in the BE-model. For example, this approach also holds for small desorption activation barriers (i.e., for $E_{\mathrm{b}}(f) \approx k_{\mathrm{B}} T$ ), and also for nonlinear barrier $v s$. force dependence. It naturally takes into account the reversible desorption-adsorption events ${ }^{36}$ which are neglected in the BEmodel. Moreover, it does not rest on the stationary approximation (which is customary in the standard Kramers rate calculation $^{24}$ ) and is, therefore, ideally suited for description of driven force-(FC) or displacement-control (DC) regimes. One of the principal results in this analytic treatment is the predicted existence of characteristic spikes, the mean force $v s$. distance profile, observed in the DC-regime. These spikes depend on the adsorption energy $\varepsilon_{\mathrm{s}}$, cantilever spring constant $k_{\mathrm{c}}$ as well as on the cantilever velocity $v_{\mathrm{c}}$. In equilibrium, this has been found earlier by Jagota and coworkers. ${ }^{17}$ The PDF of detachment forces and detachment distances are been thoroughly investigated. The relevant mean detachment force is found to be a strongly nonlinear function of $v_{\mathrm{c}}$ which is mainly governed by the nonlinear chain stretching upon increasing $v_{\mathrm{c}}$. The average full detachment (peeling) time scales $\propto 1 / v_{\mathrm{c}}$ which is supported by earlier theoretical findings. ${ }^{41}$

Some of these predictions were checked by means of MD simulations and found to be in qualitative agreement with the results, gained by the analytic method. Most notably, this applies to properties like the characteristic force oscillation pattern and the mean force $v s$. cantilever velocity $v_{\mathrm{c}}$ dependence. On the other hand, our MD simulations reveal a very strong increase in the magnitude of the force plateau for a strong-friction $\left(\gamma_{c}=25\right)$ and massive $\left(m_{c}=25\right)$ cantilever. Interestingly, in this case the spike pattern is almost totally smeared out. This might be the reason why the force spike pattern is not seen in laboratory detachment experiments. We recall that in recent Brownian dynamic simulations (which totally ignore inertial forces) ${ }^{19}$ the friction coefficient of the cantilever was 70 times larger than the friction coefficient of the chain segments. It was shown that for this high-friction cantilever and large velocity of pulling, the force spike pattern was significantly attenuated ${ }^{\mathbf{1 9}}$ so that information on the base sequence was hardly assessable. Therefore, fabrication of a stiff and super-light, nanometer-sized AFM probe would be a challenging task for future developments of biopolymer sequencing.

As an outlook, our coarse-grained Onsager stochastic model could be generalized to encompass investigations of forced unfolding of multi-domain, self-associating biopolymers. ${ }^{37}$ In doing so one should go beyond the FJBV chain model and take into account the stiffness, which is typical for most of the biopolymers.

\section{APPENDIX}

\section{A Legendre transformation}

The Gibbs free energy for the FJBV-model (i.e. the free energy in the isotensional-ensemble) has the following form

$$
G_{\mathrm{pol}}(n, \tilde{f})=-n k_{\mathrm{B}} T \ln \left[\frac{\sinh \tilde{f}}{\tilde{f}}\right],
$$

where $\tilde{f} \stackrel{\text { def }}{=} b f / k_{\mathrm{B}} T$ is the dimensionless force and $R=-\partial G_{\mathrm{pol}}(n$, $\tilde{f}) / \partial \tilde{f}$ is the corresponding distance.

$$
R=-\frac{\partial G_{\mathrm{pol}}}{\partial f}=n b \mathscr{L}(\tilde{f}),
$$

where the so-called Langevin function $\mathscr{L}(\tilde{f}) \equiv \operatorname{coth}(\tilde{f})-1 / \tilde{f}$ has been used. In the isometric-ensemble, the appropriate thermodynamic potential is the Helmholtz free energy, $F_{\text {pol }}(n, R)$, which is related to $G_{\text {pol }}(n, \tilde{f})$ by Legendre transformation,

$$
F_{\mathrm{pol}}(n, R)=G_{\mathrm{pol}}(n, \tilde{f})+f R,
$$

where $f=\partial F_{\mathrm{p}}(n, R) / \partial R$. Taking the Gibbs free energy, eqn (36), into account and the relationship eqn (37) for the Helmholtz free energy, we have

$$
\begin{aligned}
F_{\mathrm{pol}}(n, R) & =G_{\mathrm{pol}}(n, \tilde{f})+f R=-n k_{\mathrm{B}} T \ln \left[\frac{\sinh \tilde{f}}{\tilde{f}}\right]+\tilde{f} k_{\mathrm{B}} T n \mathscr{L}(\tilde{f}) \\
& =-n k_{\mathrm{B}} T\left\{\ln \left[\frac{\sinh \tilde{f}}{\tilde{f}}\right]+1-\tilde{f} \operatorname{coth}(\tilde{f})\right\} \equiv-n k_{\mathrm{B}} T \mathscr{\mathscr { C }}(\tilde{f}),
\end{aligned}
$$

where the function $\mathscr{G}(x) \equiv \ln [\sinh (x) / x]+1-x \operatorname{coth}(x)$. This finally leads to eqn (6).

\section{B Adsorption-desorption potential}

Here we prove that the adsorption-desorption potential in eqn (17) meets the conditions (1)-(4) given in Section 3.1. The minima and maxima of eqn (17) are located in the points defined by $\sin [(2 s+1) \pi]=1 / 2 \pi$ with $s$ denoting the continuous index of a monomer. As a result,

$$
s= \begin{cases}\frac{1}{2 \pi} \arcsin \left(\frac{1}{2 \pi}\right)+k-\frac{1}{2} & \text { for } k=1,2, \ldots \\ -\frac{1}{2 \pi} \arcsin \left(\frac{1}{2 \pi}\right)+n & \text { for } n=0,1,2, \ldots\end{cases}
$$

In eqn (40) the first term, $(1 / 2 \pi) \arcsin (1 / 2 \pi) \approx 0.025$, is very small and could be neglected. Thus, the minima and maxima are located at the integer and half-integer points respectively (see Fig. 4).

In order to calculate the activation barriers, we determine first $F_{\text {ads }}(s)$ at the half-integer points, i.e.,

$$
F_{\text {ads }}(n+1 / 2)=k_{\mathrm{B}} T \varepsilon(n+5 / 2)-k_{\mathrm{B}} T \varepsilon N,
$$


as well as at the integer points

$$
\begin{aligned}
& F_{\text {ads }}(n)=k_{\mathrm{B}} T \varepsilon n-k_{\mathrm{B}} T \varepsilon N \\
& F_{\text {ads }}(n+1)=k_{\mathrm{B}} T \varepsilon(n+1)-k_{\mathrm{B}} T \varepsilon N .
\end{aligned}
$$

Therefore, the activation barriers for the detachment, $\Delta E^{+}$, and adsorption, $\Delta E^{-}$, are given by

$$
\begin{aligned}
& \Delta E^{+}=F_{\text {ads }}(n+1 / 2)-F_{\text {ads }}(n)=\frac{5}{2} k_{\mathrm{B}} T \varepsilon \\
& \Delta E^{-}=F_{\text {ads }}(n+1 / 2)-F_{\text {ads }}(n+1)=\frac{3}{2} k_{\mathrm{B}} T \varepsilon
\end{aligned}
$$

i.e., $\Delta E^{+}>\Delta E^{-}$. Finally, one may readily see that $F_{\text {ads }}(0)=$ $-k_{\mathrm{B}} T \varepsilon N$ and $F_{\text {ads }}(N)=0$ which is in line with condition (4) from Section 3.1.

\section{Calculation of thermodynamic forces}

Let us calculate $f_{n} \stackrel{\text { def }}{=}-\partial \mathscr{F}(n, R) / \partial n$ for the frictionless substrate model. By making use of the free energy function, eqn (18), one has

$$
\begin{aligned}
f_{n}= & -k_{\mathrm{B}} T \varepsilon\{1-2 \pi \sin [(2 n+1) \pi]\}+k_{\mathrm{B}} T \mathscr{G}(\tilde{f}) \\
& +k_{\mathrm{B}} T n \mathscr{G}^{\prime}(\tilde{f})\left(\frac{\partial \tilde{f}}{\partial n}\right)_{R}=-k_{\mathrm{B}} T \varepsilon\{1-2 \pi \sin [(2 n+1) \pi]\} \\
& +k_{\mathrm{B}} T \mathscr{G}(\tilde{f})-\frac{k_{\mathrm{B}} T R}{b n} \frac{\mathscr{G}^{\prime}(\tilde{f})}{\mathscr{L}^{\prime}(\tilde{f})},
\end{aligned}
$$

where we have used (recall that $R / b n=\mathscr{L}(\tilde{f})$ )

$$
\left(\frac{\partial \tilde{f}}{\partial n}\right)_{R}=-\frac{R / b n^{2}}{\mathscr{L}^{\prime}(\tilde{f})}
$$

On the other hand, a direct calculation shows that

$$
\begin{aligned}
& \mathscr{G}^{\prime}(x)=\frac{x}{[\sinh (x)]^{2}}-\frac{1}{x} \\
& \mathscr{L}^{\prime}(x)=\frac{1}{x^{2}}-\frac{1}{[\sinh (x)]^{2}}
\end{aligned}
$$

so that

$$
\frac{\mathscr{S}^{\prime}(x)}{\mathscr{L}^{\prime}(x)}=-x
$$

and we arrive at the final result eqn (23).For $f_{R} \stackrel{\text { def }}{=}-\partial \mathscr{F}(n, R) / \partial R$ one obtains

$$
f_{R}=k_{\mathrm{B}} \operatorname{Tn} \mathscr{G}^{\prime}(\tilde{f})\left(\frac{\partial \tilde{f}}{\partial R}\right)_{n}+k_{\mathrm{c}}(D-R)=\frac{k_{\mathrm{B}} T}{b} \frac{\mathscr{G}^{\prime}(\tilde{f})}{\mathscr{D}^{\prime}(\tilde{f})}+k_{\mathrm{c}}(D-R)
$$

where we have used

$$
\left(\frac{\partial \tilde{f}}{\partial R}\right)_{n}=\frac{1 / b n}{\mathscr{D}^{\prime}(\tilde{f})}
$$

Taking into account eqn (46), one finally derives eqn (25).

\section{The separation $R$ as an instantaneously adjustable variable}

Due to strong adsorption, the desorbed portion of the polymer chain is expected to be strongly stretched. One could simplify the force, $f_{R} \approx(R / b n)\left[3-(R / b n)^{2}\right] /\left[1-(R / b n)^{2}\right] \approx 1 /[1-(R / b n)]$, where eqn (27) has been used and the contribution of the cantilever has been neglected. Therefore, the simplified equation which governs $R$ reads

$$
\frac{\mathrm{d} R}{\mathrm{~d} t} \approx-\frac{\lambda_{R} k_{\mathrm{B}} T}{b[1-(R / b n)]}
$$

This equation can be easily solved and the corresponding solution has the form

$$
\frac{1-R(t) / b n}{1-R_{0} / b n}=\sqrt{1+t / \tau_{R}}
$$

where the relaxation time $\tau_{R}=\left(b^{2} / 2 k_{\mathrm{B}} T \lambda_{R}\right)\left(1-R_{0} / b n\right)^{2}$. This result suggests that for a strongly stretched chain, i.e., for $R_{0} \leq$ $b n$, the relaxation time $\tau_{R}$ is very small. ${ }^{42}$ For example, in the case that $R_{0}=b(n-1)$ we have

$$
\tau_{R}=\frac{b^{2}}{2 k_{\mathrm{B}} T \lambda_{R} n^{2}}
$$

This relaxation time should be compared to the characteristic time, $\tau_{\text {kram }}$, of the slow variable $n(t)$ which is governed by the Kramers process. According to the semi-phenomenological Bell model, ${ }^{12}$ the characteristic time of unbonding (that is, desorption in our case $)$ is given by $\tau_{\text {Kram }}=\tau_{0} \exp \left[\left(\Delta E-r_{0} f_{\mathrm{p}}\right) /\right.$ $k_{\mathrm{B}} T$ ] where $\tau_{0}=\xi_{0} b^{2} / k_{\mathrm{B}} T$ is the segmental time, $\Delta E=F_{1}-F_{2}$ is the activation energy for single monomer desorption, $r_{0}$ stands for the width of adsorption potential, and $f_{\mathrm{p}}$ is the plateau height. The free energies in the desorbed, $F_{1}$, and in the adsorbed, $F_{2}$, states are given by $F_{1}=-k_{\mathrm{B}} T \ln \mu_{2}$ and $F_{2}=-k_{\mathrm{B}} T \varepsilon$ $-k_{\mathrm{B}} T \ln \mu_{3}$ where $\mu_{2}$ and $\mu_{3}$ are the so-called connective constants in two- and three dimensions respectively. ${ }^{43}$

As mentioned in Section 4.2, for large adsorption energies $\varepsilon$ the dimensionless plateau height $\tilde{f}_{\mathrm{p}} \stackrel{\text { def }}{=} b f_{\mathrm{p}} / k_{\mathrm{B}} T \propto \varepsilon$. Taking this into account, one could represent $\tau_{\text {kram }}$ in the following form:

$$
\tau_{\mathrm{Kram}}=\frac{\tau_{0} \mu_{2}}{\mu_{3}} \exp [(1-\alpha) \varepsilon]
$$

where $\alpha=r_{0} / b<1$. Therefore, in the case when $\tau_{R} \ll \tau_{\mathrm{kram}}$, the distance $R$ could be treated as the fast variable. With eqn (50) and (51) and taking into account that the Onsager coefficient $\lambda_{R}$ $=1 / \xi_{0} n$, this condition means that

$$
\frac{n \mu_{2}}{\mu_{3}} \exp [(1-\alpha) \varepsilon] \gg 1 \text {. }
$$

This condition holds for all typical values of the relevant parameters.

\section{E Runge-Kutta algorithm for stochastic differential- algebraic equations}

In order to solve the DAE (32) numerically, one may employ the second order Runge-Kutta (RK) algorithm. To this end the first equation in eqn (32) may be rewritten as an integral equation 
which relates the $i^{\text {th }}$ and $i+1$ grid points (using discrete time points $\left.t_{\mathrm{i}}=i h\right)$

$$
n_{i+1}=n_{i}+\lambda_{n} \int_{t_{i}}^{t_{i+1}} f_{n}(n(s), R(s)) \mathrm{d} s+w_{n}(h)
$$

where $h$ is the time step and $n_{\mathrm{i}}=n(i h)$. Moreover, $w_{n}(h)=\int_{t_{i}}^{t_{i+1}} \xi_{n}(s) \mathrm{d} s$ describes a Wiener process with zero mean and with variance:

$$
\begin{aligned}
\left\langle w_{n}(h) w_{n}(h)\right\rangle & =\int_{t_{i}}^{t_{i+1}} \mathrm{~d} s_{1} \int_{t_{i}}^{t_{i+1}} \mathrm{~d} s_{2}\left\langle\xi_{n}\left(s_{1}\right) \xi_{n}\left(s_{2}\right)\right\rangle \\
& =2 \lambda_{n} k_{\mathrm{B}} T \int_{t_{i}}^{t_{i+1}} \mathrm{~d} s_{1} \int_{t_{i}}^{t_{i+1}} \mathrm{~d} s_{2} \delta\left(s_{1}-s_{2}\right)=2 \lambda_{n} k_{\mathrm{B}} T h
\end{aligned}
$$

The integral over the deterministic force in eqn (53) within this $2^{\text {nd }}$ order approximation reads

$$
\int_{t_{i}}^{t_{i+1}} f_{n}(n(s), R(s)) \mathrm{d} s \approx \frac{h}{2}\left[f_{n}\left(n_{i+1}, R_{i+1}\right)+f_{n}\left(n_{i}, R_{i}\right)\right]+\mathscr{O}\left(h^{3}\right)
$$

This is so-called trapezoidal rule for approximation of the integral. In order to calculate $f_{n}\left(n_{i}, R_{i}\right)$, one should first take the initial value $n_{i}$, and find $R_{i}$ through the solution of the nonlinear equation $G\left(n_{i}, R_{i}, t_{i}\right)=0$. For the calculation of $f_{n}\left(n_{i+1}, R_{i+1}\right)$, one can use the forward Euler method of order 1 , i.e., $n_{i+1}^{\mathrm{E}}=n_{i}+h \lambda_{n} f_{n}\left(n_{i}, R_{i}\right)+h^{1 / 2}\left(2 \lambda_{n} k_{\mathrm{B}} T\right)^{1 / 2} Z_{n}$ and $R_{i+1}^{\mathrm{E}}$ are obtained as solution of the equation $G\left(n_{i+1}^{\mathrm{E}}, R_{i+1}^{\mathrm{E}}, t_{i+1}\right)=0$. Here the random variable $Z_{n}$ is Gaussian with the zero mean value and with variance

$$
\left\langle Z_{n}{ }^{2}\right\rangle=1
$$

As a result, the recursive procedure which relates the $i$-th and $(i+1)$-th grid points can be defined as:

1. For a given initial value of $n_{i}$, go to eqn (29) or eqn (30) and solve this nonlinear equation (e.g. $\left.G\left(n_{i}, R_{i}, t_{i}\right)=0\right)$ with respect to $R_{i}$.

2. Compute $g_{1}=f_{n}\left(n_{i}, R_{i}\right)$.

3. Compute $n_{i+1}$ and $R_{i+1}$ within the Euler approximation, i.e., calculate first $n_{i+1}^{\mathrm{E}}=n_{i}+h \lambda_{n} f_{n}\left(n_{i}, R_{i}\right)+h^{1 / 2}\left(2 \lambda_{n} k_{\mathrm{B}} T\right)^{1 / 2} Z_{n}$ and then solve $G\left(n_{i+1}^{\mathrm{E}}, R_{i+1}^{\mathrm{E}}, t_{i+1}\right)=0$ with respect to $R_{i+1}^{\mathrm{E}}$.

4. Compute $g_{2}=f_{n}\left(n_{i+1}^{\mathrm{E}}, R_{i+1}^{\mathrm{E}}\right)$.

5. Compute the corrected $n_{i+1}$, i.e.

$$
n_{i+1}=n_{i}+\frac{h}{2}\left(g_{1}+g_{2}\right)+h^{1 / 2}\left(2 \lambda_{n} k_{\mathrm{B}} T\right)^{1 / 2} Z_{n}
$$

6. Finally, with the value of $n_{i+1}$, go to item 1 and solve the nonlinear equation $G\left(n_{i+1}, R_{i+1}, t_{i+1}\right)=0$ with respect to $R_{i+1}$.

\section{Acknowledgements}

This work was supported by grant SFB 625 from German Research Foundation (DFG). Computational time on PL-Grid Infrastructure is acknowledged. A.M. thanks the Max Planck Institute for Polymer Research in Mainz, Germany, and CECAMMPI for hospitality during his visit at the Institute.

\section{References}

1 F. Ritort, J. Phys.: Condens. Matter, 2006, 18, R531-R583.

2 I. Franco, M. A. Ratner and G. C. Schatz, Single-Molecule Pulling: Phenomenology and Interpretation, in Nano and Cell Mechanics: Fundamentals and Frontiers, ed. H. D. Esinosa and G. Bao, Wiley, Microsystem and Nanotechnology Series, 2013, ch. 14, pp. 359-388.

3 B. N. Balzer, M. Gallei, M. V. Hauf, M. Stallhofer, L. Wiegleb, A. Holleiner, M. Rehahn and T. Hugel, Angew. Chem., Int. Ed., 2013, 52, 6541-6544.

4 H.-J. Butt, B. Cappella and M. Kappl, Surf. Sci. Rep., 2005, 59, $1-152$.

5 Y. Gao, G. Sirinakis and Y. Zhang, J. Am. Chem. Soc., 2011, 133, 12749-12757.

6 A. Janshof, M. Neitzert, Y. Oberdorfer and H. Fuchs, Angew. Chem., Int. Ed., 2000, 39, 3212-3237.

7 M. Carrion-Vazquez, A. F. Oberhauser, S. Fowler, P. E. Marszalek, S. E. Broedel, J. Clarke and J. M. Fernandez, Proc. Natl. Acad. Sci. U. S. A., 1999, 96, 3694-3699.

8 T. E. Fisher, A. F. Oberhauser, M. Carrion-Vazquez, P. E. Marszalek and J. M. Fernandez, Trends Biochem. Sci., 1999, 24, 379-384.

9 H. Clausen-Schaumann, M. Seitz, R. Krautbauer and H. E. Gaub, Curr. Opin. Chem. Biol., 2000, 4, 524-530.

10 C. E. Sing and A. Alexander-Katz, Macromolecules, 2011, 44, 6962-6971.

11 C. E. Sing and A. Alexander-Katz, Macromolecules, 2012, 45, 6704-6718.

12 G. I. Bell, Science, 1978, 200, 618-627.

13 E. Evans and K. Ritchie, Biophys. J., 1997, 72, 1541-1555.

14 E. Evans, Annu. Rev. Biophys. Biomol. Struct., 2001, 30, 105128.

15 E. Evans and D. A. Calderwood, Science, 2007, 316, 11481153.

16 R. Merkel, P. Nassoy, K. Ritchi and E. Evans, Nature, 1999, 397, 50-53.

17 S. Manohar and A. Jagota, Phys. Rev. E: Stat., Nonlinear, Soft Matter Phys., 2010, 81, 021805.

18 S. Manohar, A. R. Manz, K. E. Bancroft, Ch-Y. Hui, A. Jagota and D. V. Vezenov, Nano Lett., 2008, 8, 4365-4372.

19 S. Iliafar, D. Vezenov and A. Jagota, Langmuir, 2013, 29, 14351444.

20 S. Iliafar, K. Wagner, S. Manohar, A. Jagota and D. Vezenov, J. Phys. Chem. C, 2012, 116, 13896-13903.

21 H. J. Kreuzer, S. H. Payne and L. Livadaru, Biophys. J., 2001, 80, 2505-2514.

22 D. B. Staple, F. Hanke and H. J. Kreuzer, Phys. Rev. E: Stat., Nonlinear, Soft Matter Phys., 2008, 77, 021801.

23 D. B. Staple, M. Geisler, T. Hugel, L. Kreplak and H. J. Kreuzer, New J. Phys., 2011, 13, 013025.

24 P. Hänggi, P. Talkner and M. Borcovec, Rev. Mod. Phys., 1990, 62, 251-341.

25 F. Hanke and H. J. Kreuzer, Phys. Rev. E: Stat., Nonlinear, Soft MatterPhys., 2006, 74, 031909. 
26 S. Dattagupta and S. Puri, Dissipative Phenomena in Condensed Matter, Springer-Verlag, Berlin, 2004.

27 S. Bhattacharya, V. G. Rostiashvili, A. Milchev and T. A. Vilgis, Macromolecules, 2009, 42, 2236-2250.

28 S. Bhattacharya, V. G. Rostiashvili, A. Milchev and T. A. Vilgis, Phys. Rev. E: Stat., Nonlinear, Soft Matter Phys., 2009, 79, 030802(R).

29 S. Bhattacharya, A. Milchev, V. G. Rostiashvili and T. A. Vilgis, Eur. Phys. J. E, 2009, 29, 285-297.

30 Y.-J. Sheng and P.-Y. Lai, Phys. Rev. E: Stat. Phys., Plasmas, Fluids, Relat. Interdiscip. Top., 1997, 56, 1900-1909.

31 J. U. Schurr and S. B. Smith, Biopolymers, 1990, 29, 11611165.

32 A. Serr and R. R. Netz, Europhys. Lett., 2006, 73, 292-298.

33 J. Paturej, A. Milchev, V. G. Rostiashvili and T. A. Vilgis, Macromolecules, 2012, 45, 4371-4380.

34 A. Cohen, Rheol. Acta, 1991, 30, 270-273.
35 U. M. Ascher and L. R. Petzold, Computer Methods for Ordinary Differential Equations and Differential-Algebraic Equations, SIAM, Philadelphia, 1998.

36 G. Diezemann and A. Janshoff, J. Chem. Phys., 2008, 129, 084904.

37 D. B. Staple, S. H. Payne, A. L. C. Reddin and H. J. Kreuzer, Phys. Biol., 2009, 6, 025005.

38 K. Eom, D. E. Makarov and G. J. Rodin, Phys. Rev. E: Stat., Nonlinear, Soft Matter Phys., 2005, 71, 021904.

39 G. Yoon, S. Na and K. Eom, J. Chem. Phys., 2012, 137, 025102.

40 F. Li and D. Leckband, J. Chem. Phys., 2006, 125, 194702.

41 U. Seifert, Phys. Rev. Lett., 2000, 84, 2750-2753.

42 M. Febbo, A. Milchev, V. Rostiashvili, D. Dimitrov and T. A. Vilgis, J. Chem. Phys., 2008, 129, 154908.

43 C. Vanderzande, Lattice Models of Polymers, Cambridge University Press, Cambridge, 1998. 